\title{
HYDRODYNAMIC LIMITS FOR ONE-DIMENSIONAL PARTICLE SYSTEMS WITH MOVING BOUNDARIES ${ }^{1}$
}

\author{
By L. Chayes and G. Swindle \\ University of California at Los Angeles and University of California \\ at Santa Barbara
}

\begin{abstract}
We analyze a new class of one-dimensional interacting particle systems featuring random boundaries with a random motion that is coupled to the local particle density. We show that the hydrodynamic limiting behavior in these systems corresponds to the solution of an appropriate Stefan (free-boundary) equation and describe some applications of these results.
\end{abstract}

\section{Introduction.}

Overview and preliminaries. The study of interacting particle systems with conservation laws frequently revolves around hydrodynamic limits, in which the long-time, large-length scale behavior of a system is characterized by the evolution of the local density of the conserved quantity according to a partial differential equation. The typical setup consists of a particle system defined on a closed lattice with periodic boundary conditions or, perhaps, with a fixed particle density at the boundary. See, for example, [13] and references therein for an extensive discussion of these topics. In each of these cases, the dynamics of the particle system is prescribed by the transition rates, the initial condition and, if relevant, the boundary conditions. In particular, the internal dynamics of the particle systems does not affect the behavior of the boundaries.

In this paper we study certain one-dimensional particle systems with exclusion dynamics and the additional feature that the region in which exclusion dynamics occurs is altered by the dynamics itself. The two basic examples can both be regarded as crude microscopic models of the dynamics of a liquid-solid system with an interface: the first case corresponds to the melting of a solid and the second to the freezing of a supercooled liquid.

To describe the process of melting, consider the following particle system with configurations in $\{-1,0,1\}^{\mathbb{L}_{N}}$, where $\mathbb{L}_{N}=\{-N,-N+1, \ldots, N-1, N\}$.

Received September 1994; revised June 1995.

${ }^{1}$ Work supported in part by the NSF under Grants DMS-93-02023 (L.C.) and DMS-93-05904 (G.S.).

AMS 1991 subject classifications. 60K35, 60H15.

Key words and phrases. Particle systems, exclusion process, Stefan's equation. 
We will denote the configuration at time $t$ by $\zeta_{t}$ and the state of site $i$ at time $t$ by $\zeta_{t}(i)$. As usual, we think of sites $i$ for which $\zeta_{t}(i)=1$ as being occupied by particles and when $\zeta_{t}(i)=0$, the site is vacant. When $\zeta_{t}(i)=-1$, the state is quite different from either of the above. Let us regard these sites as being occupied by land mines or (static) antiparticles. The transition rates are described as follows: any nearest-neighbor pair of sites in which one is occupied and the other is vacant exchange their states at unit rate. Note that this rule refers only to the exchanges among 0's and 1's, for example, $(\ldots 01 \ldots) \rightarrow(\ldots 10 \ldots)$. The rule for antiparticles is that any neighboring pair of sites in which one is occupied and the other is a mine will become a pair of vacant sites at unit rate: $(\ldots-11 \ldots) \rightarrow(\ldots 00 \ldots)$. All other transitions involving the antiparticles are suppressed. Let us consider this process with the initial configuration satisfying

$$
\zeta_{0}(i)= \begin{cases}-1, & \text { if }-N \leq i \leq 0 \\ 0 \text { or } 1, & \text { otherwise }\end{cases}
$$

in which all sites to the left of 1 are antiparticles and all sites to the right of 0 are vacant or occupied. It is clear that among the antiparticles, the first transition does not occur until the leftmost 1 jumps onto the mine at the origin. They "annihilate," vacating both the sites at 0 and at 1 . Subsequent annihilation transitions continue to erode the region of -1 's while further reducing the number of particles. With the above labeling, the block of -1 's may be thought of as the cold reservoir of a melting block and the +1 particles as corpuscles of heat. Alternatively, exchanging labels among 0's and 1's, each -1 is revealed to be a frozen pair of particles that decouple, at unit rate, whenever space is available. In any case, as the block melts, more room is made available for dynamics.

Quite the opposite behavior is observed at the water-ice interface of a supercooled liquid. Here the boundary moves inward, encroaching on the fluid region. We model this system on the same space as before, $\{-1,0,+1\}^{\mathbb{L}_{N}}$, and, similarly, the transitions among the 0's and 1's are given by the usual rules for the simple exclusion process. Furthermore, the -1 's are again essentially static, but this time, new -1's can be generated. First, we instill the rule that any configurations in which $\mathrm{a}+1$ neighbors $\mathrm{a}-1$ is forbidden. (This statement is true, by fiat, in the initial configuration and is dynamically enforced thereafter.) The dynamics among +1's and -1 's may now be described as follows: when a +1 exchanges with a 0 that neighbors a -1 , during the course of the exchange, the +1 is transformed into a -1 . Explicitly, the transitions $(\ldots-1 \quad 0 \quad 1 \ldots) \rightarrow(\ldots-1-1 \quad 0 \ldots)$ and $(\ldots 10-1 \ldots) \rightarrow(\ldots 0-1-1 \ldots)$ occur at unit rate.

These two processes will now be defined precisely. For any finite interval $\mathbb{M} \subset \mathbb{Z}$, it is sufficient to specify the action of the infinitesimal generator, $\Omega_{\mathbb{M}}$, on any real-valued function on the configuration space $\{-1,0,+1\}^{\mathbb{M}}$. Starting with the first (melting) process, let $\zeta \in\{1,0,+1\}^{\mathbb{M}}$ denote a particle configu- 
ration and, for $i$ and $j$ in $\mathbb{M}$, we define the possible $i \rightarrow j$ particle transfer configurations by

$$
\begin{aligned}
& \zeta^{[i ; j]}(k)=\zeta(k), \quad k \neq i, k \neq j, \\
& \zeta^{[i ; j]}(j)= \begin{cases}\zeta(j)+1, & \zeta(j)<1 \text { and } \zeta(i)=1, \\
\zeta(j), & \text { otherwise, }\end{cases} \\
& \zeta^{[i ; j]}(i)= \begin{cases}0, & \zeta(i)=1 \text { and } \zeta(j)<1, \\
\zeta(i), & \text { otherwise. }\end{cases}
\end{aligned}
$$

If $\partial \mathbb{M}$ denotes the endpoints of $\mathbb{M}$ and $\mathbb{M}^{\circ}=\mathbb{M} \backslash \partial \mathbb{M}$, we define the generator

$$
\Omega_{\mathbb{M}} f(\zeta)=\sum_{i \in \mathbb{M}^{\circ}}\left[f\left(\zeta^{[i-1 ; i]}\right)+f\left(\zeta^{[i+1 ; i]}\right)-2 f(\zeta)\right]+\Omega_{\partial \mathbb{M}} f
$$

where $\Omega_{\partial \mathbb{M}}$ is a generator for describing particle transfers to the endpoints that will depend on the particulars of the problem.

To distinguish the second (freezing) process from the first, we will use $\eta$ to denote the particle configuration and $\mathscr{L}_{M}$ to denote the generator. The action of the generator is otherwise exactly the same as in (1.3a), but here the $i \rightarrow j$ particle transfer configurations are given by

$$
\begin{aligned}
& \eta^{[i ; j]}(k)=\eta(k), \quad k \neq i, k \neq j, \\
& \eta^{[i ; j]}(j)= \begin{cases}+1, & \text { if } \eta(i)=1, \eta(j)=0 \text { and } \eta(j \pm 1) \neq-1, \\
-1, & \text { if } \eta(i)=1, \eta(j)=0 \text { and } \eta(j-1)=-1 \\
\eta(j), & \text { or } \eta(j+1)=-1,\end{cases} \\
& \eta^{[i ; j]}(i)= \begin{cases}0, & \eta(i)=1 \text { and } \eta(j) \neq 1, \\
\eta(i), & \text { otherwise. }\end{cases}
\end{aligned}
$$

To simplify the notation in much of what is to follow in later sections, for appropriately smooth functions $g(\zeta ; t)$ [or, informally, $g\left(\zeta_{t} ; t\right)$ ] that depend explicitly on time as well as the "instantaneous" configuration $\zeta_{t} \in$ $\{-1,0,+1\}^{\mathbb{M}}$, we will use $\Upsilon_{\mathbb{M}}$ to denote the evolution operator:

$$
\Upsilon_{\mathbb{M}} g\left(\zeta_{t} ; t\right)=\lim _{\varepsilon \rightarrow 0^{+}} \frac{1}{\varepsilon}\left[\mathbf{E}\left(g\left(\zeta_{t+\varepsilon} ; t+\varepsilon\right) \mid \zeta_{t}\right)-g\left(\zeta_{t} ; t\right)\right] .
$$

Similarly, for the freezing $(\eta)$ problem we will use the notation $\mathscr{Y}_{\mathbb{M}}$.

REMARK. Provided that $|\mathbb{M}|<\infty$, the objects $\Omega_{\mathbb{M}}$ and $\mathscr{L}_{\mathbb{M}}$ are clearly sufficient to specify the process. For a system defined on all of $\mathbb{Z}$, more work would be required (cf. [11], Chapter 1, for a discussion of these matters), but for our purposes, such a result would hardly be worth the effort. Hydrodynamic limits most often involve $|\mathbb{M}| \rightarrow \infty$ rather than starting on a system that is infinite from the outset. Furthermore, as particle systems per se, these 
two examples are not so interesting to study. In both cases, the invariant measures would have a tendency to be trivial - at best, one of the invariant measures for the simple exclusion process.

As is well known, the hydrodynamic limit of the usual exclusion process is the (linear) heat equation. Thus, it is not hard to imagine that the hydrodynamic limit of the above processes are Stefan equations in which the diffusive evolution governs both the relaxation of the internal density as well as the motion of the free boundary.

The classical form of the (one-sided) Stefan equation for the density $\rho(x, t)$ and the free boundary $B(t)$ is given by

$$
\frac{\partial \rho}{\partial t}=\frac{\partial^{2} \rho}{\partial x^{2}},
$$

subject to a possible (fixed) boundary condition at, for example, $x=x_{0}$, an initial condition on $B$ and an initial condition on the density:

$$
\begin{aligned}
B(0) & =B_{0}, \\
\rho\left(x_{0}, t\right) & =\rho_{F}(t), \\
\rho(x, 0) & =\rho_{0}(x), \quad B_{0} \leq x \leq x_{0} .
\end{aligned}
$$

We may assume that all of the above is taking place for $0 \leq t \leq T$ where, for simplicity, we have $T<\infty$. The boundary condition at the moving boundary is, typically,

$$
\rho(B(t), t)=0,
$$

and, finally, we arrive at the so-called Stefan condition that relates the evolution of $B$ to the flux of $\rho$ through $B$. The most often studied version of the Stefan equation (subject to a myriad of generalizations) has, as the Stefan condition,

$$
\frac{d B}{d t}=-\nabla \rho(B(t), t) .
$$

Clearly, this corresponds to the outward displacement of the boundary (melting) and is the type of limiting behavior that one would expect from the particle system defined in (1.2) and (1.3). The other possibility is

$$
\frac{d B}{d t}=+\nabla \rho(B(t), t)
$$

(and its generalizations) corresponding to an inward displacement (freezing) at the boundary. One would expect this sort of limiting behavior from the particle system described in (1.5). Of course, since we have described the Stefan problem in classical terms, all of the preceding equations are subject to the stipulation that the various quantities are well defined; in practice, one usually deals with the weak forms of (1.5)-(1.8).

The systems governed by the type of Stefan condition in (1.8a) are far more tractable than those systems corresponding to (1.8b); the vast majority of 
the work on Stefan's equation concerns the first case and its generalizations. The reason for this discrepancy can be understood from the perspective of the enthalpy. Since this quantity will enter directly into our analysis, it is worthwhile to describe the differences between the two systems in these terms.

Formally (or in the weak version) both systems may be expressed as a diffusion equation for an enthalpy function, $a(x, t)$, with a diffusion coefficient that depends discontinuously on the value of $a$. In both cases, we have $a(x, t)=\rho(x, t)$ for $x>B(t)$; however, for the system described in (1.8a), the relevant definition is

$$
a(x, t)=-1 \text { if } x<B(t),
$$

while in the case of the system described in (1.8b), one defines

$$
a(x, t)=+1 \text { if } x<B(t) .
$$

In the former case, the diffusion coefficient is a monotone nondecreasing function of the enthalpy while, in the latter case, it is not. With the benefit of this monotonicity, uniqueness of the Stefan system - in quite some generality -is immediate. However, without this monotonicity, the problem is far more challenging. (In particular, the system is borderline ill-posed and has a definite potential for instabilities.) In fact, the existing literature on the subject turns out to be insufficient for our purposes.

Returning the discussion to the particle systems, it is intuitively clear that in the first system, the enthalpy corresponds to $\zeta$ itself while in the second, the enthalpy corresponds to $|\eta|$. In both cases, an appropriate (martingale) version of the weak form of the relevant Stefan equation is readily derived. In the former case, the desired hydrodynamic limit is then an immediate consequence of the established uniqueness results for this equation. (The precise notion of convergence will appear in the statement of Theorem 2.2.) In the latter case, general results on uniqueness are not known and hence must be established here. Even so, our results require the a priori knowledge that the boundary is a continuous function of time. In fact, continuity of the boundary is not a direct consequence of the existence of a weak solution to this version of Stefan's equation. It therefore must be directly established in the context of the particle system. This is somewhat nontrivial (when one considers diffusive time scaling) and constitutes a major portion of our efforts. However, once these ingredients have been assembled, a hydrodynamic limit is readily established for this case as well. (The precise notion of convergence will appear in the statement of Theorem 3.4.)

Organization. The organization of this paper is as follows. In Section 2, we analyze the problem with melting boundaries. Using the standard derivations in the theory of hydrodynamic limits, we readily arrive at a weak form of the appropriate Stefan equation. The well-known uniqueness results for this version of the equation provide us with the final stages of the hydrodynamic limit in this case. 
In Section 3, we start the procedure along the same lines (with the analysis of Section 2 allowing us to avoid most of the calculations). Thus, any hydrodynamic limit satisfies a weak version of the appropriate Stefan equation. To maneuver into a position where uniqueness results can be brought to bear, continuity of the rescaled boundaries is established in a series of detailed arguments. Using the results from the Appendix, the rest of the hydrodynamic limit is straightforward.

In Section 4, we discuss an application of a variant of the system studied in Section 3: the dynamical disappearance of a two-dimensional droplet that is trapped against the corner of a sample. The appropriate extensions of the analysis in Section 3 are briefly performed.

In the Appendix, we provide the missing ingredients for the proofs of the results in Sections 3 and 4, namely, a proof of uniqueness for the solutions of Stefan equations with inwardly moving boundaries under the hypothesis of continuous boundaries.

2. Analysis of a system with a melting boundary. Consider the particle system on $\mathbb{L}_{N}$ with bulk dynamics as described in (1.2) and (1.3). To complete the definition of the system, we must describe the behavior at the endpoints. At the right end, we will force the system into a prescribed (deterministic) particle density. Let $R_{F}(t)$ denote a (piecewise) continuous function with $0 \leq R_{F}(t) \leq 1$. For the site at $k=N$ and for $i \in \mathbb{L}_{N}^{\circ}$, we define $\zeta^{[N ; i]}$ as before. Let

$$
\begin{aligned}
& \zeta(k)^{[N ; \infty]}=\zeta(k) \text { for } k \neq N, \\
& \zeta^{[N ; \infty]}(N)= \begin{cases}0, & \text { if } \zeta(N)=1, \\
\zeta(N), & \text { otherwise, }\end{cases}
\end{aligned}
$$

denote the configuration where the site at $N$ has just shipped out a particle and let

$$
\begin{aligned}
\zeta(k)^{[\infty ; N]} & =\zeta(k) \text { for } k \neq N, \\
\zeta^{[\infty ; N]}(N) & = \begin{cases}\zeta(N)+1, & \text { if } \zeta(N)<1, \\
\zeta(N)(\equiv 1), & \text { otherwise, }\end{cases}
\end{aligned}
$$

denote the configuration where the site at $N$ has just received one. Similar notions apply at the left boundary (or, in general, to any other site). For the problem at hand, we define

$$
\begin{aligned}
\Omega_{\partial \mathbb{L}_{N}} f=\left[1-R_{F}(t)\right]\left[f\left(\zeta^{[N ; \infty]}\right)-f(\zeta)\right]+R_{F}(t)\left[f\left(\zeta^{[\infty ; N]}\right)-f(\zeta)\right] \\
+\left[f\left(\zeta^{[N-1 ; N]}\right)-f(\zeta)\right]+\left[f\left(\zeta^{[-N+1 ;-N]}\right)-f(\zeta)\right] .
\end{aligned}
$$

The result of this action, at the right endpoint, will be to push the density toward $R_{F}(t)$. (At the left end, for completeness, we have installed a boundary condition that would enforce zero gradient at $k=-N$. Needless to say, this will be practically irrelevant: our initial configuration will place $k=-N$ 
deep inside a frozen block and the process itself ceases to be of interest just when the first +1 particle has made its way to this point.)

In the continuum, a weak version of the Stefan equation with receding boundaries is formulated as follows: suppose, without any significant loss of generality, that the spatial domain is $[-1,+1]$ and that the initial position of the boundary is $B(0)=0$. We will assume that at $x=1$, the density is fixed at $\rho_{F}(t)$ which is piecewise smooth and satisfies $0 \leq \rho_{F}(t) \leq 1$. For $1 \geq x \geq 0$, we will take the initial configuration to be given by some $\rho_{0}(x)$ which is also piecewise smooth and bounded between 0 and 1 . Let $G(x, t)$ denote any smooth test function with $G(1, t) \equiv 0$. Then, the enthalpy function $a(x, t)$ satisfies

$$
\begin{aligned}
\int_{-1}^{+1}[ & a(x, s) G(x, s)-a(x, 0) G(x, 0)] d x \\
& =\int_{-1}^{+1} \int_{0}^{s}\left[a \frac{\partial G}{\partial t}+H(a) \frac{\partial^{2} G}{\partial x^{2}}\right] d x d t-\int_{0}^{s} \rho_{F}(t) \frac{\partial G}{\partial x}(1, t) d t
\end{aligned}
$$

where

$$
H(a)= \begin{cases}0, & \text { if } a \leq 0 \\ a, & \text { if } a \geq 0\end{cases}
$$

REMARK. Formally, (2.4) is a nonlinear diffusion equation with the diffusion coefficient given by $D(a)=H^{\prime}(a)$. The case of the single-phase Stefan problem can also be treated by the introduction of an auxiliary density, $n(x)$, which enters into $(2.4 \mathrm{a})$ as the coefficient of $g_{x x}$. We will see that the formulation using $n(x)$ is slightly more convenient for this section, while in the next section it is actually necessary because of a spurious ambiguity in the formulation of the problem as a nonlinear diffusion equation.

The boundary, $B(t)$, in either formulation is simply

$$
B(t)=\sup \{x \mid a(x, t)=-1\},
$$

which, as it turns out, is well defined for each $t$ (cf. the discussion in Proposition 2.1). It also turns out that in the solution to this system, the boundary moves continuously to the left until some time $T$ [which depends on the functions $\rho_{F}(t)$ and $\rho_{0}(x)$ ] when it hits the point $x=-1$. In the present formulation, the solution may be continued-if $\rho_{F}(t)$ has been definedbut thereafter, it is an ordinary diffusion problem on $[-1,+1]$ with a zerogradient boundary condition at $x=-1$.

For this particle system, the local enthalpy is the value of $\zeta$ at each site and the auxiliary density is the positive part thereof. We formally define

$$
a_{t}(k)=\zeta_{t}(k)
$$

and

$$
n_{t}(k)=\left[\zeta_{t}(k)\right]^{+}
$$


Further, if $y \in[0, N]$ is a real number, we will write

$$
a_{t}(y)=a_{t}(k(y))
$$

for the $k(y)$ that satisfies $k-\frac{1}{2}<y \leq k+\frac{1}{2}$ and similarly for $n_{t}(y)$. In this and in the later sections, we will be interested in convergence to the hydrodynamic limit via a sequence of systems that double in size at each stage. We thus start with some $N_{0}$ that is arbitrary (but large enough so that all quantities under discussion make sense) and we define, for $r$ a positive integer, $N_{r}=2^{r} N_{0}$. Of primary interest will be the function

$$
a_{r}(x, t)=a_{N_{r}^{2} t}\left(N_{r} x\right)
$$

and

$$
n_{r}(x, t)=n_{N_{r}^{2} t}\left(N_{r} x\right),
$$

with $-1 \leq x \leq+1$ and $0 \leq t \leq T$. Our first substantive result will concern the expected values of these quantities.

Proposition 2.1. Let $S>0$ denote any positive time and let $\rho_{F}(t)$ and $\rho_{0}(x)$ be defined as above with $0 \leq t \leq S$. Consider the particle systems on $\mathbb{L}_{N_{r}}$ as described in (1.2), (1.3) and (2.1)-(2.3) with $R_{F}(t)$ given by $\rho_{F}\left(N_{r}^{2} t\right)$. Suppose, further, that each realization of the particle system comes equipped with an initial particle configuration that is, deterministically, $\zeta_{0}(k)=-1$ for $k \leq 0$ while, for $0<k \leq N_{r}$, the $\zeta_{0}(k)$ are independent with $\mathbf{E}\left[\zeta_{0}(k)\right]=$ $\rho_{0}(k / N)$. Let $\bar{a}_{r}(x, t)=\mathbf{E}\left[a_{r}(x, t)\right]$ denote the expected value of the enthalpy in the rth particle system. Then

$$
\bar{a}_{r}(x, t) \rightarrow a(x, t),
$$

weakly in $L^{2}$, where $a(x, t)$ is the (unique) solution to the Stefan problem described in (2.4).

REMARK. The fact that the weak limit is uniquely determined is of no immediate consequence-it is just a spin-off of the known uniqueness results for this system. What will actually be proved is that the limit of any converging subsequence of $\left(a_{r}\right)$ is a weak solution to the system described in (2.4).

Let $g_{t}(k)$ denote any (deterministic) function on $\mathbb{L}_{N}$ which, for all $t$ and $k$, is differentiable with respect to $t$. Define $\langle g, a\rangle_{t}$ by

$$
\langle g, a\rangle_{t}=\sum_{k} g_{t}(k) a_{t}(k) .
$$

It is clear that

$$
\Upsilon_{\mathbb{L}_{N}}\langle g, a\rangle_{t}=\left\langle\frac{\partial g}{\partial t}, a\right\rangle_{t}+\left\langle g, \Omega_{\mathbb{L}_{N}} a\right\rangle_{t},
$$

where the notation in (2.10) is defined analogously to that in (2.9). 
In the interior, $\Omega_{\mathbb{L}_{N}}$ acts in exactly the same fashion as the generator for the usual exclusion process; however, the behavior at the boundaries requires special consideration. We define the microscopic boundary as the location of the antiparticle that is farthest to the right:

$$
b_{r}(t)=-\left[N_{r}+1\right]+\sum_{k \geq-N_{r}} \delta_{\left[\zeta_{t}(k),-1\right]},
$$

where $\delta_{\left[\zeta_{t}(k),-1\right]}$ indicates the presence of an antiparticle at the site $k$. The rules for $\Omega_{\mathbb{L}_{N}} a_{t}(k)$ are easily discerned from the definition of the process:

$$
\begin{gathered}
\Omega_{\mathbb{L}_{N}} a_{t}(k)=0, \quad k<b_{r}(t), \\
\Omega_{\mathbb{Q}_{N}} a_{t}\left(b_{r}(t)\right)=a_{t}\left(b_{r}(t)+1\right), \\
\Omega_{\mathbb{L}_{N}} a_{t}\left(b_{r}(t)+1\right)=a_{t}\left(b_{r}(t)+2\right)-2 a_{t}\left(b_{r}(t)+1\right), \\
\Omega_{\mathbb{Q}_{N}} a_{t}(k)=a_{t}(k+1)+a_{t}(k-1)-2 a_{t}(k) \equiv \Delta a_{t}(k), \\
\Omega_{\mathbb{L}_{N}} a_{t}(N)=R_{F}(t)+a_{t}(N-1)-2 a_{t}(N) .
\end{gathered}
$$

We thus have

$$
\left\langle g, \Omega_{\mathbb{L}_{N}} a\right\rangle_{t}=g_{t}\left(b_{t}\right) a_{t}\left(b_{t}+1\right)+g_{t}\left(b_{t}+1\right)\left[a_{t}\left(b_{t}+2\right)-2 a_{t}\left(b_{t}+1\right)\right]
$$

$$
\begin{aligned}
& +\sum_{k=b_{t}+2}^{N-1} g_{t}(k) \Delta a_{t}(k) \\
& +g_{t}(N)\left[R_{F}(t)+a_{t}(N-1)-2 a_{t}(N)\right] .
\end{aligned}
$$

Defining $g_{t}(N+1) \equiv 0$ and performing the usual summation by parts, we obtain

$$
\begin{aligned}
\left\langle g, \Omega_{\mathbb{L}_{N}} a\right\rangle_{t}= & \sum_{k=b_{t}+1}^{N} a_{t}(k) \Delta g_{t}(k) \\
& -R_{F}(t)\left[g_{t}(N+1)-g_{t}(N)\right],
\end{aligned}
$$

where the dummy $g_{t}(N+1)$ is included for the ease of future reference. Since, for $k>b_{t}, a_{t}(k)=n_{t}(k)$ and, for $k \leq b_{t}, n_{t}(k)=0$, it is seen that the first term on the right-hand side of (2.14) can be expressed as $\langle n, \Delta g\rangle_{t}$. We can now conclude that

$$
\begin{aligned}
m_{r}(g, t)= & \langle g, a\rangle_{t}-\langle g, a\rangle_{0}-\int_{0}^{t}\left(\left\langle\frac{\partial g}{\partial t}, a\right\rangle_{s}+\langle\Delta g, n\rangle_{s}\right) d s \\
& +\int_{0}^{t} R_{F}(t)\left[g_{t}(N+1)-g_{t}(N)\right] d s
\end{aligned}
$$


is a martingale with zero expectation. Let $G(x, t)$ be a smooth test function that vanishes at $x=1$ and define, for $s \leq N_{r}^{2} S$,

$$
g_{s}(k)=G\left(\frac{k}{N_{r}+1}, \frac{s}{N_{r}^{2}}\right) \text {. }
$$

Notice that $g_{s}$ is now defined on $\{-N-1, \ldots, N+1\}$. We may formally extend $a_{s}(k)$ to this domain by defining $a_{s}(N+1)=R_{F}(s)$ and, for example, $a_{s}(-N-1)=0$.

Using the notation

$$
M_{r}(G)=\frac{1}{N_{r}} m_{r}\left(G\left(\frac{k}{N_{r}+1}, \frac{s}{N_{r}^{2}}\right)\right)
$$

and recalling the quantities $a_{r}$ and $n_{r}$ from the definition prior to the statement of this lemma, we find that

$$
\begin{aligned}
M_{r}(G)= & \int_{-1}^{+1}\left[G(x, t) a_{r}(x, t)-G(x, 0) a_{r}(x, 0)\right] d x \\
& +\int_{0}^{t} d s \rho_{F}(s) \frac{\partial G}{\partial x}(1, s) \\
& -\int_{0}^{t} d s \int_{-1}^{+1}\left(a_{r}(x, s) \frac{\partial G}{\partial t}+n_{r}(x, s) \frac{\partial^{2} G}{\partial x^{2}}\right) d x+O\left(\frac{1}{N_{[r]}}\right) .
\end{aligned}
$$

Averaging over both sides of (2.17), we have, modulo terms of the order of $N_{r}^{-1}$, the weak form of the Stefan equation. Hence, any convergent subsequence converges to a weak solution of this system.

The uniqueness of solutions to (2.4) is well established; an elegant derivation can be found in [7]. Furthermore, under the stated initial and boundary conditions, the solution is classical and $B(t)$ is $C^{\infty}$ for positive times.

REMARK. Any reader who has checked the details of the calculations leading to (2.14) will notice a fortuitous cancellation of terms at the microscopic boundary $b_{t}$. Indeed, had we defined the rates for the switches $(\ldots-11 \ldots) \rightarrow(\ldots 00 \ldots)$ to be $\kappa$ instead of unity, there would have been an unwanted term of the form

$$
u_{t} \propto[1-\kappa] a_{t}\left(b_{t}+1\right)\left[g_{t}\left(b_{t}+1\right)-g_{t}\left(b_{t}\right)\right] .
$$

Note that, in the hydrodynamic limit, this does not vanish due to power counting alone: indeed, we get one factor of $N_{r}^{-1}$ in the transition from $m_{r}(g)$ to $M_{r}(G)$ and another because we have on display, essentially, $N_{r}^{-1}$ times the gradient of $G$. However, there are also of the order of $N_{r}^{2}$ time steps involved in the integration, so ostensibly we could end up with a factor of the order of unity. Nevertheless, under diffusive scaling, this term exerts no influence (provided that $\kappa>0$ ). Presumably, this is due to the (a posteriori) fact that the density at the moving boundary-here represented by the term $a_{t}\left(b_{t}+1\right)$-vanishes in the hydrodynamic limit. We have not been able to 
implement this directly into an argument but the following is unquestionably related.

Let us assume, for simplicity, that $g_{t}(k)$ is time independent. (The effects of time dependence in $g$ indeed fall to naive power counting.) Observe that the unwanted term admits the expression

$$
u_{t} \propto \frac{1}{\kappa} \Omega_{\mathbb{L}_{N}} \sum_{k \leq b_{t}} a_{t}(k) \nabla g(k) .
$$

Thus, in expectation, the time integral of this quantity is equal to the argument of the generator evaluated at the endpoints:

$$
\mathbf{E}\left[\int_{0}^{N_{r}^{2} t} u_{s} d s\right] \propto \mathbf{E}\left(\sum_{k \leq b_{N_{r}^{2} t}} a_{N_{r}^{2} t}(k) \nabla g(k)-\sum_{k \leq b_{0}} a_{0}(k) \nabla g(k)\right) .
$$

Since the $a$ 's are constants in both the sums, the gradient may be summed and the right-hand side is just $g$ evaluated at the endpoints. Thus, at this stage, the unwanted term is of the order of unity and then there is the additional factor of $N_{r}^{-1}$ in the passage from $m_{r}(-)$ to $M_{r}(-)$.

The final argument of this section requires only one more set of calculations.

Theorem 2.2. Let $a_{r}(x, t)$ be as described in Proposition 2.1. Then, with probability 1 ,

$$
a_{r}(x, t) \rightarrow a(x, t),
$$

weakly in $L^{2}$, where $a(x, t)$ is the solution to the Stefan problem described in (2.4) with the stated initial and boundary conditions.

PROOF. For any smooth $G$, we have established that the random variables $M_{r}(G)$ have expected values that are bounded by inverse powers of $N_{r}$. If the same can be established for $M_{r}^{2}$, then due to the rapid growth of the $\left(N_{r}\right)$, we have that $M_{r}(G)$ converges to 0 with probability 1 . But this implies that all weakly convergent subsequences of the random $a_{r}(x, t)$ converge to the solution $a(x, t)$ which directly implies the stated result.

We proceed with the quadratic variation calculation which, except for the action at the boundary, is fairly standard in the study of hydrodynamic limits.

Since $M_{r}$ is of the form $W-\int \Upsilon W d t$, the quadratic variation is equal to $\Upsilon W^{2}-2 W \Upsilon W$. It is easily seen that we may ignore the constant $(t=0)$ term. Writing

$$
\langle g, a\rangle_{t}^{2}=\sum_{j, k} a_{t}(j) a_{t}(k) g_{t}(j) g_{t}(k),
$$

it is found that all terms involving time derivatives of $g$ drop out of $\Upsilon_{\mathbb{L}_{N}}\left[\langle a, g\rangle_{t}\right]^{2}-2\langle a, g\rangle_{t} \Upsilon_{\mathbb{L}_{N}}\langle a, g\rangle_{t}$. Furthermore, for any $j$ and $k$ that satisfy 
$\Omega_{\mathbb{L}_{N}} a_{t}(j) a_{t}(k)=a_{t}(j) \Omega_{\mathbb{L}_{N}} a_{t}(k)+a_{t}(k) \Omega_{\mathbb{L}_{N}} a_{t}(j)$, we get a cancellation. This leaves only the diagonal and near-diagonal terms:

$$
\begin{aligned}
& N_{r}^{2} \Upsilon_{\mathbb{L}_{N}} M_{r}^{2}=\sum_{k \in \mathbb{L}_{N}} g_{t}^{2}(k)\left[\Omega_{\mathbb{L}_{N}} a_{t}^{2}(k)-2 a_{t}(k) \Omega_{\mathbb{L}_{N}} a_{t}(k)\right] \\
& +\sum_{k: k, k-1 \in \mathbb{L}_{N}} g_{t}(k) g_{t}(k-1)\left[\Omega_{\mathbb{L}_{N}}\left(a_{t}(k) a_{t}(k-1)\right)\right. \\
& -a_{t}(k) \Omega_{\mathbb{a}_{N}} a_{t}(k-1) \\
& \left.-a_{t}(k-1) \Omega_{\mathbb{L}_{N}} a_{t}(k)\right] \\
& +\sum_{k: k, k+1 \in \mathbb{L}_{N}} g_{t}(k) g_{t}(k+1)\left[\Omega_{\mathbb{L}_{N}}\left(a_{t}(k) a_{t}(k+1)\right)\right. \\
& -a_{t}(k) \Omega_{\mathbb{L}_{N}} a_{t}(k+1) \\
& \left.-a_{t}(k+1) \Omega_{\mathbb{L}_{N}} a_{t}(k)\right] \text {. }
\end{aligned}
$$

After a lengthy calculation, with special attention to the boundary terms, the following results emerge: the second and third summations of (2.22) (which are identical after reindexing) each lead to

$$
\begin{aligned}
& -\sum_{k=b_{t}+1}^{N-1}\left[g_{t}(k) g_{t}(k+1)\left(a_{t}(k)-a_{t}(k+1)\right)^{2}\right] \\
& \quad-a_{t}\left(b_{t}+1\right) g_{t}\left(b_{t}\right) g_{t}\left(b_{t}+1\right),
\end{aligned}
$$

while the first summation on the right-hand side of (2.22) equals

$$
\begin{aligned}
& \sum_{k=b_{t}+1}^{N-1}\left[g_{t}^{2}(k)+g_{t}^{2}(k+1)\right]\left[a_{t}(k+1)-a_{t}(k)\right]^{2} \\
& \quad+a_{t}\left(b_{t}+1\right)\left[g_{t}^{2}\left(b_{t}\right)+g_{t}^{2}\left(b_{t}+1\right)\right] \\
& \quad+g_{t}^{2}(N)\left[R_{F}+a_{t}(N)-2 R_{F} a_{t}(N)\right] .
\end{aligned}
$$

Combining these two lines, we finally arrive at

$$
\begin{aligned}
\Upsilon_{\mathbb{L}_{N_{r}}} M_{r}^{2}(G)= & \frac{1}{N_{r}^{2}} \sum_{k=b_{t}+1}^{N-1}\left(a_{t}(k)-a_{t}(k+1)\right)^{2}\left[g_{t}(k)-g_{t}(k+1)\right]^{2} \\
& +\frac{a_{t}\left(b_{t}+1\right)}{N_{r}^{2}}\left[g_{t}\left(b_{t}\right)-g_{t}\left(b_{t}+1\right)\right] \\
& +\frac{g_{t}^{2}(N)}{N_{r}^{2}}\left[R_{F}+a_{t}(N)-2 R_{F} a_{t}(N)\right] .
\end{aligned}
$$

Integrating the right-hand side from 0 to $N_{r}^{2} t$, we see that $\mathbf{E}\left[M_{r}^{2}(G)\right]$ vanishes at least as rapidly as $N_{r}^{-1}$. 
REMARK. Note that the contributions from the moving boundary and the static boundary were both of the order of $N_{r}^{-2}$ [in the latter case because $\left.g_{t}(N) \sim 1 / N\right]$. Oddly enough, in the calculation of the quadratic variation, a different jump rate at the moving boundary would only have generated a term of this order and therefore would not have required a separate argument as in the remark following Proposition 2.1.

3. Analysis of a system with a freezing boundary. In this section, we will take up the analysis of the particle system described in (1.4) and (1.3). As for the boundary generators, at $x=N$, we will use the same device as in the previous section [cf. (2.1) and (2.2) and the relevant parts of (2.3)]. We will always start the process with $\eta_{0}(-N)=-1$; thus, as can be seen from the dynamics, no boundary terms will be necessary on this side. (In the next section, we will discuss some problems in which the moving boundaries are placed on both sides of $\mathbb{L}_{N}$; in these cases, there will be essentially no need for a boundary generator.)

In this case, the continuum description presents a few difficulties. If one attempts to describe this system as a nonlinear diffusion problem for the enthalpy, it is clear that this quantity must be defined according to (1.9b) with an $H(a)$ given by

$$
H(a)= \begin{cases}a, & \text { if } a \leq 1, \\ 1, & \text { if } a \geq 1 .\end{cases}
$$

However, this forces $D(a)=0$ whenever $a \geq 1$ while, from the classical description, there is no reason to suppose that the enthalpy does not exceed 1 to the right of the boundary. Even in the particle system, a region of $a=1$ could represent (mobile) particles packed at unit density. This is not an insurmountable problem in our particular case because these situations can only occur in the initial condition and will disappear the instant that the process starts. However, in closely related particle models - for example, in which the boundary moves more than one lattice spacing for each particle that it adsorbs - the same problem will crop up and, perhaps, cannot simply be defined away. Therefore, in principle, we should work with the auxiliary density form of the weak equation which reads:

$$
\begin{aligned}
& \int_{-1}^{+1}[a(x, s) G(x, s)-a(x, 0) G(x, 0)] d x \\
& \quad=\int_{-1}^{+1} \int_{0}^{s}\left[a(x, t) \frac{\partial G}{\partial t}+n(x, t) \frac{\partial^{2} G}{\partial x^{2}}\right] d x d t-\int_{0}^{s} \rho_{F}(t) \frac{\partial G}{\partial x}(1, t) d t .
\end{aligned}
$$

Note that, in general, it takes the combination of both $a$ and $n$ to determine the nature of the solution. This is in sharp contrast to the weak equation featured in the previous section.

As discussed previously, far less is known about the solution to (3.2) than the solution to (2.4); in particular, there the boundary is not a priori a well-defined object. On the other hand, suppose there is a solution to (3.2) in 
which $B(t)$ [defined, e.g., as the boundary of the region where $n(x, t)=a(x, t)$ ] is known to be a continuous function. Then $n(x, t)$ satisfies the equation

$$
\begin{aligned}
& \int_{B(t)}^{1} n(x, t) G(x) d x-\int_{B(0)}^{1} \rho_{0}(x) G(x) d x \\
& \quad=\int_{0}^{t} d s \int_{B(s)}^{1} n(x, s) \frac{\partial^{2} G}{\partial x^{2}}-\int_{0}^{t} \frac{\partial G}{\partial x}(1) \rho_{F}(s) d s-\int_{B(0)}^{B(t)} G(x) d x,
\end{aligned}
$$

where, for simplicity, we have assumed that the test function is time independent. With some mild restrictions on $\rho_{0}(x)$ and $\rho_{F}(t)$, it is possible to show that only one pair $(n(x, t), B(t))$ exists that satisfies this equation. This is the subject of the Appendix, which is not particularly short and, from the perspective of particle systems, not particularly enlightening.

For the particle system under study in this section, it is straightforward to show that the weak (hydrodynamic) limits satisfy (3.2) -indeed, this is just a recapitulation of the derivations in Proposition 2.1 and Theorem 2.2. The key ingredient in this section is therefore a proof of continuity of the boundary $B(t)$. This is the subject of Proposition 3.3. Our final result-the analog of Theorem 2.2 -is then a fairly straightforward corollary to all the above mentioned.

Proposition 3.1. Consider the particle systems on $\mathbb{\mathbb { L }}_{N}$ as described in (1.4) and (1.3) with boundary generator at $k=+N$ as described in (2.1), (2.2) and the relevant portion of (2.3). Let

$$
n_{t}(k)=\left[\eta_{t}(k)\right]^{+}
$$

and

$$
a_{t}(k)=\left|\eta_{t}(k)\right|
$$

Let us define functions $a_{r}(x, t)$ and $n_{r}(x, t)$ as in (2.7) and (2.8) and let $\bar{a}_{r}$ and $\bar{n}_{r}$ denote the expected values of these quantities. Then $(\bar{a}, \bar{n})$ is a solution to the (Stefan) equation (3.2), where $(\bar{a}, \bar{n})$ denotes any weak limit of $\left(\bar{a}_{r}, \bar{n}_{r}\right)$. Furthermore, with probability 1 , if $\left(a^{\star}(x, t), n^{\star}(x, t)\right)$ is any weak $L^{2}$ limit of the (random) sequence $\left(a_{r}, n_{r}\right)$, then $\left(a^{\star}(x, t), n^{\star}(x, t)\right)$ also satisfies this equation.

Proof. We follow closely the previous derivations. For technical convenience, here and in the remainder of this section, we will define the microscopic boundary as the position of the hole that is just to the right of the region of frozen particles:

$$
b_{r}(t)=-N_{r}+\sum_{k \geq-N_{r}} \delta_{\left(\eta_{t}(k),-1\right)}
$$

[so that we may still write $\eta_{t}\left(b_{t}+1\right) \propto \mathscr{L}_{\mathbb{I}_{N}} b_{t}$ ]. Mimicking exactly the steps in (2.9) to (2.16), we arrive, unimpeded, at the analog of (2.17). [The remark following the proof of Proposition 2.1 applies here as well; here, the unwanted boundary terms take the form $(\kappa-1)\left[g_{t}\left(b_{r}\right)-g_{t}\left(b_{r}+1\right)\right] \eta_{t}\left(b_{r}+1\right)$ and can 
be handled by the same argument.] The stated result for $\left(\bar{a}_{r}, \bar{n}_{r}\right)$ follows immediately.

The second portion of this proposition is another quadratic variation calculation for the analog of the martingale $M_{r}(G)$. (Here we will use the same notation for the corresponding object.) The result of an identical procedure is exactly (2.23) [with the $b_{r}(t)$ as given by (3.3) above].

Since the $N_{r}$ are so sparse, this implies $M_{r}(G) \rightarrow 0$ with probability 1 . Let $\left(a_{r}, n_{r}\right)$ denote a single realization and let $\left(a^{\star}, n^{\star}\right)$ denote a weak $L^{2}$ subsequential limit of $\left(a_{r}, n_{r}\right)$. Note that, ostensibly, $\left(a^{\star}, n^{\star}\right)$ is random. Let $G_{\alpha}$ denote a countable collection of test functions (with the appropriate boundary condition) that are dense in $L^{2}$. Since, with probability 1 , for all $\alpha, M_{r}\left(G_{\alpha}\right) \rightarrow 0$ along the subsequence, this implies $\left(a^{\star}, n^{\star}\right)$ is a weak solution to the Stefan equation in the sense of (3.2).

REMARK. Insofar as Proposition 3.1 is concerned, we can be fairly cavalier about the nature of the boundary and the initial conditions. For the sake of (ultimately) obtaining classical results, we will assume that $\rho_{0}(x)$ and $\rho_{F}(t)$ are piecewise continuous. Henceforth, we will also assume that $\rho(x, 0)$ does not go to 0 too fast at $x=-1$ or to 1 too fast at $x=+1$. Explicitly, we assume that there are constants $w<\infty$ and $v>0$ such that

$$
\rho_{0}(x) \leq w(1+x)
$$

and

$$
\rho_{0}(x) \leq 1-v(1-x) .
$$

With a certain amount of additional labor, all of the above can be relaxed-although some condition is needed that prevents $\rho_{0}(x) \equiv 1$ in a neighborhood of $x=-1$.

We now attend to the behavior of the boundaries. The starting point will be a lemma concerning a quantity that also plays a central role in the continuum analysis of the Stefan equation (in the Appendix) as well as in the applications.

DeFINITION. Let

$$
\Delta_{N}(t)=\sum_{-N}^{+N} a_{t}(k)[N-k]
$$

denote the displacement in the particle configuration $\eta_{t}$. Observe that if the initial configuration has $a_{t}(k) \equiv 0$, then $\Delta_{N}(t)$ is exactly how much total leftward motion has occurred in the particle system. The correct (diffusive) scaling for this object is seen to be $\left(1 / N^{2}\right) \Delta_{N}\left(N^{2} t\right)$. Thus, we further define

$$
\delta_{r}(t)=\frac{1}{N_{r}^{2}} \Delta_{N_{r}}\left(N_{r}^{2} t\right) .
$$


Lemma 3.2. Let $\delta_{r}(t)$ be as defined above and let $\mathscr{Q}_{F}(t)=\int_{0}^{t} \rho_{F}(s) d s$. Then $\delta_{r}(t) \rightarrow \delta(t)$, in the sup norm, with probability 1 , where

$$
\delta(t)= \begin{cases}\delta(0)+\mathscr{Q}_{F}(t), & \mathscr{Q}_{F}(t) \leq 2-\delta(0), \\ 2, & \mathscr{Q}_{F}(t) \geq 2-\delta(0) .\end{cases}
$$

REMARK. This is essentially a repeat of the analysis in [2]; for completeness, a brief derivation will be included.

Proof of Lemma 3.2. The configuration $\eta_{t}$ can be naturally divided into a number, $K_{t}(\eta)$, of distinct clumps of +1 particles. [However, we will adopt the convention that if $\eta_{t}(N)=1$, we will not count the rightmost clump in our calculation of $K_{t}$.] The quantity $\Delta_{N}(t)$ changes by \pm 1 each time a particle goes forward or backward, and such events only take place at the endpoints of the clumps or at the rightmost (non)clump of the system. Let $\eta_{t}$ denote any configuration in which $\Delta_{N}<2 N(N-1)$ (i.e., $\left.b_{t}<N\right)$-so there are still some dynamics. Each clump represents the chance to increase $\Delta_{N}(t)$ by one unit at unit rate. In addition, if $\eta_{N}(t)=0$, a new particle will be introduced into the system at rate $R_{F}$ and otherwise there is the final uncounted clump that allows for one additional opportunity to increase $\Delta_{N}(t)$ (by one unit, at unit rate). Evidently, under the condition $b_{t}<N$,

(3.5a) $\Delta_{N}(t) \rightarrow \Delta_{N}(t)+1$ at rate $K_{t}+\eta_{t}(N)+\left(1-\eta_{t}(N)\right) R_{F}$.

Similarly,

$$
\Delta_{N}(t) \rightarrow \Delta_{N}(t)-1 \text { at rate } K_{t}+\eta_{t}(N)\left(1-R_{F}\right) .
$$

The above may be expressed, succinctly, as

$$
\mathscr{L}_{\mathbb{L}_{N}} \Delta_{N}(t)=R_{F}(t) \mathbb{1}_{b_{t}<N} .
$$

To remove the (unwanted) indicator in (3.6), let us define $\tau_{N}=\sup \left\{t \mid b_{t}<N\right\}$ and

$$
D_{N}(t)= \begin{cases}\Delta_{N}(t), & t \leq \tau_{N}, \\ 2 N(N-1)+\int_{\tau_{N}}^{t} R_{F}(s) d s, & t \geq \tau_{N} .\end{cases}
$$

It is thus seen that the quantity $D_{N}(t)-\left(\mathscr{Q}_{F}(t)+\Delta_{N}(0)\right)$ is a martingale. Let us compute the variance. It is clear that if $t>\tau_{N}$, then $\mathscr{Y}_{\mathbb{L}_{N}} D_{N}(t)=2 D_{N} R_{N}$. For $t<\tau_{N}$, an easy calculation shows

$$
\mathscr{Y}_{\mathbb{L}_{N}} \Delta_{N}^{2}\left(t ; t<\tau_{N}\right)=2 R_{F} \Delta_{N}(t)+2 K_{t}+2 \eta_{t}(N)\left(1-R_{F}\right)+R_{F} .
$$

Of course, $2 R_{F} \Delta_{N}(t)$ is what we anticipate for deterministic motion. The final few terms involving $R_{F}$ are insignificant, so the only term of potential significance is $2 K_{t}(\eta)$.

We claim, however, that in any configuration, $\Delta_{r}(t) \geq \frac{1}{2}\left(K_{t}\right)\left(K_{t}-1\right)$. Indeed, let $p_{m}, m=1,2, \ldots, K_{t}$, denote the displacement of the particle at the 
left end of the $m$ th clump - counting from the left. It is clear that to the right of the $m$ th clump, there must be at least $m-1$ holes. Thus, we have $p_{m} \geq(m-1)$ and hence $\Delta_{r}(t) \geq \sum_{m} p_{m}=\frac{1}{2}\left(K_{t}\right)\left(K_{t}-1\right)$. Using the bound

$$
\mathbf{E}\left[\sqrt{D_{N}(t)-D_{N}(0)}\right] \leq \sqrt{E\left[D_{N}(t)-D_{N}(0)\right]} \leq \tilde{c} t^{1 / 2},
$$

we can average and integrate (3.7) to obtain

$$
\mathbf{E}\left(D_{N}^{2}\right)-\mathbf{E}\left[\left(D_{N}\right)\right]^{2} \leq c t^{3 / 2}
$$

where, in the above, $c$ and $\tilde{c}$ are constants of order unity. Defining $d_{r}(t) \equiv$ $D_{N_{r}}\left(N_{r}^{2} t\right) / N_{r}^{2} t$ and so on, (3.8) easily implies

$$
d_{r}(t) \rightarrow d(t)
$$

pointwise, with probability 1 . Let $t^{\star}$ denote the anticipated end of the simulation

$$
2=\delta(0)+\int_{0}^{t^{\star}} \rho_{F}(s) d s .
$$

Choosing a time greater than $t^{\star}$, say $2 t^{\star}$, and using Doob's inequality (modified suitably for a continuous-time process that, with probability 1 , has only a finite number of jumps), one obtains

$$
\operatorname{Prob}\left[\sup _{t \leq 2 t^{\star}}\left|d_{r}(t)-d(t)\right|>\varepsilon\right] \leq \frac{\tilde{c}^{\prime}\left[t^{\star}\right]^{3 / 2}}{\varepsilon^{2} N_{r}} .
$$

The above equation readily implies convergence in the sup norm, with probability 1 and, incidentally, that $\tau_{N_{r}} / N_{r}^{2} \rightarrow t^{\star}$.

Next we show that limiting boundaries produced by the particle system are continuous functions of time.

Proposition 3.3. Let $B_{r}(t)$ denote the random boundary rescaled so as to be of order unity and expressed as a function of rescaled time:

$$
B_{r}(t)=\frac{1}{N_{[r]}} b_{r}\left(N_{[r]}^{2} t\right) .
$$

Then, for any infinite sequence $r_{\bar{k}}$, there is, with probability 1 , a further (ostensibly random) subsequence $r_{k}$ such that $B_{r_{k}}(t)$ converges to some limiting (ostensibly random) function $B(t)$ that is monotone and continuous.

PRoOF. Our strategy can be broken down into three steps. First, we make use of Lemma 3.2 to show that in any particular interval of time, the overall transport to the left is not unreasonably large. Next we show that, with high probability, the particle density at the boundary is bounded away from unity. In our final step, we combine the above two ingredients: in essence, we allow all the available particles to use all the available transport to create as much boundary as possible in the allowed time. After rescaling, what emerges is (except for minor details) a statement of Hölder continuity with exponent $\frac{1}{2}$. 
To keep the equations reasonable, we will shift everything to the right by $N$ units - so that the process now takes place on the lattice sites $\{0,1, \ldots, 2 N\}$-and we will assume that the $B$ boundary starts out at 0 . Let $B_{r_{b}}(q)$ denote any subsequence that converges pointwise to some $B(q)$ for all rational $q$ in $\left[0, t^{\star}\right]$. Obviously, $B(q)$ is monotone. If we can establish that $B(q)$ is continuous, then [since each $B_{r}(t)$ is monotone] we will have that $B_{r_{k}}(t) \rightarrow B(t)$.

Let $\varepsilon$ denote any positive rational that is not too large and recall the quantity $v$ that was defined in the remark following Proposition 3.1. We will show that, with probability 1 ,

$$
B(q+\varepsilon)-B(q)<\frac{2}{v(2-B(q))} \sqrt{\varepsilon}+o(\sqrt{\varepsilon})
$$

holds for all rational $q$ 's in $\left[0, t^{\star}\right)$ such that the left-hand side is defined. By (countable) subadditivity, the above can be achieved by working with an arbitrary rational $q$ in $\left[0, t^{\star}\right)$; thus, let $q$ be any rational in $\left[0, t^{\star}\right)$. Recall the displacement variable $\Delta_{N_{r}}(t)$ and the quantity $\mathscr{Q}_{F}(t)$ from the preceding lemma. We claim that, with probability 1 ,

$$
\begin{aligned}
& \Delta_{N_{r}}\left([q+\varepsilon] N_{r}^{2}\right)-\Delta_{N_{r}}\left(q N_{r}^{2}\right) \\
& \quad<\varepsilon N_{r}^{2}+\mathscr{Q}_{F}\left([q+\varepsilon] N_{r}^{2}\right)-\mathscr{Q}_{F}\left(q N_{r}^{2}\right) \leq 2 \varepsilon N_{r}^{2}
\end{aligned}
$$

holds for all but a finite number of $r$ 's. (The expected value of the left-hand side is the middle term without the $\varepsilon N_{r}^{2}$; the rightmost inequality comes from the fact that $\rho_{F} \leq 1$.)

Our next task is to put a cap on the density in the immediate vicinity of the boundary. Let $H$ be a number of order unity, the precise value of which will be determined later; let $h=H \sqrt{\varepsilon} N_{r}$ and let

$$
\mathscr{T}(h, v, b)=h-v h\left(2-\frac{b}{N}\right)+\frac{v}{N} h^{2} .
$$

Our midrange goal will be a demonstration that

$$
\mathbf{P}\left[\sum_{p=1}^{h} \eta_{t}\left(b_{r}(t)+p\right)>\mathscr{T}\left(h, v, b_{t}\right)\right] \leq \gamma_{1}(H) \exp \left[-\gamma_{2}(H) \varepsilon N_{r}\right],
$$

where $\gamma_{1}<\infty$ and $\gamma_{2}>0$ are constants independent of $b_{r}$. We remark in passing that if the above can be established independently of $t$, then, by fixing $t=N_{r}^{2} q$ at this (rescaled) time, with probability 1 , the stated event occurs for only finitely many values of $r$.

Let us start by pointing out that

$$
\begin{aligned}
\sum_{k=b_{t}}^{b_{t}+h}\left[1-v\left(2-\frac{k}{N}\right)\right] & \approx h-v h\left(2-\frac{b_{t}}{N}\right)+\frac{1}{2} \frac{v}{N} h^{2} \\
& \equiv \mathscr{T}\left(h, v, b_{t}\right)-\frac{1}{2} \frac{v}{N} h^{2}
\end{aligned}
$$


is, on the average, what would be observed for a problem with a linear density profile that has unit density at $k=2 N$ and a slope of $v / N$. This is, of course, by design; such a system will be constructed and used as a comparison. Explicitly, let us consider the simple exclusion process on

$$
\left\{-\left[2 N\left(\frac{1-v}{v}\right)\right],-\left[2 N\left(\frac{1-v}{v}\right)\right]+1, \ldots, 2 N\right\}
$$

with the left-end boundary locked at zero density and the right end at unit density. Let $\eta_{0}$ be the initial configuration where, without loss of generality, we will set $\eta_{0}(0)=-1$ and assume that $\eta_{0}(k) \geq 0, \forall k \geq 0$. Let $\eta_{t}$ denote the configuration at time $t$ and let $\eta_{t}^{l, v}$ denote the configuration starting at $\left[\eta_{0}\right]^{+}$evolving under the usual rules for the exclusion process on $\{-[2 N((1-v) / v)], \ldots, 2 N\}$ with the aforementioned boundary conditions. By coupling according to the scheme where the lattice sites (rather than the particles) are encoded with jump instructions, it is clear that, for any $k$ and at any time, $\eta_{t}^{l, v} \geq \eta_{t}(k)$. Hence, if $\mathbf{E}_{\mathrm{St}}^{\eta_{0}}(-)$ and $\mathbf{E}_{l, v}^{\eta_{0}}(-)$ denote expectations in these processes and $F(\eta)$ is any nondecreasing function of (all of) its arguments, it is clear that

$$
\mathbf{E}_{\mathrm{St}}^{\eta_{0}}(F) \leq \mathbf{E}_{l, v}^{\eta_{0}}(F) .
$$

Thus, we have, pathwise, that the event described in (3.14) is more likely in the $(l, v)$ process than in the Stefan system regardless of what $b_{r}(t)$ would have turned out to be. However, the question of the value of $b_{r}(t)$ may prove to be a nuisance, so we will circumvent this issue by being incredibly wasteful. We write

$$
\begin{aligned}
\mathbf{P}_{\mathrm{St}}^{\eta_{0}}[ & \left.\sum_{p=1}^{h} \eta_{t}\left(b_{r}(t)+p\right)>\mathscr{T}\left(h, v, b_{t}\right)\right] \\
& =\sum_{b} \mathbf{P}_{\mathrm{St}}^{\eta_{0}}\left[\left\{\sum_{p=1}^{h} \eta_{t}(b+p)>\mathscr{T}(h, v, b)\right\} \cap\left\{b_{r}(t)=b\right\}\right] \\
& \leq \sum_{b} \mathbf{P}_{\mathrm{St}}^{\eta_{\eta_{0}}}\left[\sum_{p=1}^{h} \eta_{t}(b+p)>\mathscr{T}(h, v, b)\right] \\
& \leq \sum_{b} \mathbf{P}_{l, v}^{\eta_{0}}\left[\sum_{p=1}^{h} \eta_{t}(b+p)>\mathscr{T}(h, v, b)\right] .
\end{aligned}
$$

Of course, for any fixed $b$, we have

$$
\begin{aligned}
\mathbf{P}_{l, v}^{\eta_{0}}\left[\sum_{p=1}^{h} \eta_{t}(b+p)>\mathscr{T}(h, v, b)\right] \\
\quad \leq \exp [-\alpha \mathscr{T}(h, v, b)] \mathbf{E}_{l, v}^{\eta_{0}}\left[\exp \left(\alpha\left[\sum_{p=1}^{h} \eta_{t}(b+p)\right]\right)\right] .
\end{aligned}
$$


We will estimate the final term in (3.17) by using duality. The following argument is based on results found in [11]; similar techniques are used in [5]. The dual model to the $(l, v)$ system is also the exclusion process on $\{-[2 N((1-v) / v)], \ldots, 2 N\}$ with the additional feature that particles are absorbed at the left and right boundaries. Let us denote these fictitious boundary points (which can house an indefinite number of particles) by $l^{*}$ and $r^{*}$. Starting with $j$ particles in the dual model, let us label these particles [1], [2], .., [j] and use $\hat{\eta}_{t}^{[k]}$ to denote the location of the $k$ th particle at time $t$. By convention, if for some $s<t$, the $k$ th particle is absorbed, for example, at the right, we will say $\hat{\eta}_{t}^{[k]}=r^{*}$. The dual relationship between these models may be expressed as follows: we denote by $\sigma_{t}^{[k]}\left(\eta_{0}\right)$ the event that, at time $t$, the $k$ th particle is on a site that was occupied in the configuration $\eta_{0}$ or is resting at $r^{*}$. Then, for any $A \subset$ $\{-[2 N((1-v) / v)], \ldots, 2 N\}$,

$$
\mathbf{E}_{l, v}^{\eta_{0}}\left[\prod_{k \in A} \eta_{t}(k)\right]=\hat{\mathbf{E}}_{l, v}^{A}\left[\prod_{k=1}^{|A|} \mathbb{1}_{\sigma_{t}^{[k]}\left(\eta_{0}\right)}\right],
$$

where $\hat{\mathbf{E}}_{l, v}^{A}(-)$ denotes expectation with respect to the dual process starting with initial configuration $A$.

In the case that $A$ is a singleton, as $t \rightarrow \infty$, the right-hand side is given by the probability that a random walk starting at $k$ hits the right side before it hits the left- this is the origin of the linear density profile in the stationary measure. For $|A|>1$, on the basis of [9] and [10], it turns out that the right-hand side can be bounded by the expectation of the same function with respect to the measure associated with a system of independent particles that, in all other respects, behaves identically to the interacting system. This inequality can be derived by following, step by step, the derivation in [11], Chapter 8, Proposition 1.7, modifying, when necessary, for the presence of boundary conditions. Thus, so far, we may write

$$
\begin{aligned}
\mathbf{E}_{l, v}^{\eta_{0}}\left(\eta_{t}\left(k_{1}\right) \cdots \eta_{t}\left(k_{j}\right)\right) & \leq \hat{\mathbf{E}}_{l, v}^{\mathscr{F} ; A}\left[\prod_{k_{m} \in A} \mathbb{1}_{\sigma_{t}^{\left[k_{m}\right]}\left(\eta_{0}\right)}\right] \\
& \equiv \prod_{m=1}^{j} \hat{\mathbf{E}}_{l_{, v}}^{\left\{k_{m}\right\}}\left[\mathbb{1}_{\sigma_{t}\left(\eta_{0}\right)}\right],
\end{aligned}
$$

where $A=\left\{k_{1}, \ldots, k_{j}\right\}$ and the $\mathscr{F}$ signifies expectation with respect to the distribution of the independent particle process.

We have nearly achieved the midrange goal. However, to get the estimate in (3.14), we need to average the right-hand side of (3.18) over all initial configurations $\eta_{0}$ and it appears that the individual factors in the final term in (3.20) are tangled by the initial configurations. Nevertheless, we claim that, after this averaging over the initial configurations - in which there are 
no correlations in the distribution of particles-what emerges is the product of the individual averages. Explicitly, we claim that

$$
\left\langle\prod_{m=1}^{j} \hat{\mathbf{E}}_{l, v}^{\left\{k_{m}\right\}}\left[\mathbb{1}_{\sigma_{t}\left(\eta_{0}\right)}\right]\right\rangle_{\rho_{0}}=\prod_{m=1}^{j} \bar{\eta}_{t}\left(k_{m}\right),
$$

where $\langle-\rangle_{\rho_{0}}$ denotes the average overrealizations of the initial configurations $\eta_{0}$ in which the particles are independently delivered according to $\mathbf{P}\left(\eta_{0}(k)=\right.$ $1)=\rho_{0}(k / N)$ and $\bar{\eta}_{t}(k)$ is the particle density [in the $(l, v)$ system] averaged over time and initial conditions. It is, of course, noted, by duality, that $\bar{\eta}_{t}(k)=\left\langle\mathbf{E}_{l, v}^{\langle k\}}\left[\mathbb{1}_{\sigma_{t}\left(\eta_{0}\right)}\right]\right\rangle_{\rho_{0}}$. Let us perform the configurational average of the right-hand side of (3.20) before we take the average over the time evolution. Working in the ensemble of the $j$ noninteracting particles, we condition on the locations $\hat{\eta}_{t}^{[1]}, \ldots, \hat{\eta}_{t}^{[j]}$ and average over the manifestly independent initial particle densities at these sites. Performing the time average, we thus obtain

$$
\left\langle\hat{\mathbf{E}}_{l, v}^{\mathscr{F} ; A}\left[\prod_{k_{m} \in A} \mathbb{1}_{\sigma_{t}^{\left[k_{m}\right]}\left(\eta_{0}\right)}\right]\right\rangle_{\rho_{0}}=\hat{\mathbf{E}}_{l, v}^{\mathscr{F} ; A}\left[\prod_{k_{m} \in A} \tilde{\sigma}_{t}^{\left[k_{m}\right]}\left(\bar{\eta}_{0}\right)\right],
$$

where $\tilde{\sigma}_{t}^{\left[k_{m}\right]}\left(\bar{\eta}_{0}\right)=\bar{\eta}_{0}(k)\left[\equiv \rho_{0}(N / k)\right]$ if $\hat{\eta}_{t}^{\left[k_{m}\right]}=k$ and equals 1 or 0 if $\hat{\eta}_{t}^{\left[k_{m}\right]}=$ $r^{*}$ or $l^{*}$, respectively. Obviously, the right-hand side of (3.22) factors and the resulting terms are of the stated form.

Notice that the initial density is smaller than the linear profile that represents the asymptotic density: $\bar{\eta}_{0}(k) \leq 1+v(k / N-2)$. It therefore follows that for all times $\bar{\eta}_{t}(k) \leq 1+v(k / N-2) \equiv \bar{\eta}_{\infty}(k)$. We finally arrive at

$$
\begin{aligned}
\left\langle\mathbf{E}_{l, v}^{\eta_{0}}\left(\eta_{t}\left(k_{1}\right) \ldots \eta_{t}\left(k_{j}\right)\right)\right\rangle_{\rho_{0}} & \leq \prod_{m=1}^{j} \bar{\eta}_{\infty}\left(k_{m}\right) \\
& =\prod_{m=1}^{j}\left[1+v\left(\frac{k_{m}}{N}-2\right)\right],
\end{aligned}
$$

where in the above it is assumed that the points $k_{1}, \ldots, k_{j}$ are distinct. An immediate consequence of (3.23) is the exponential estimate:

$$
\begin{aligned}
\left\langle\mathbf{E}_{l, v}^{\eta_{0}}\left[\exp \left(\alpha\left[\sum_{p=1}^{h} \eta_{t}(b+p)\right]\right)\right]\right\rangle_{\rho_{0}} & \leq \prod_{p=1}^{h}\left[1+\alpha(\exp \alpha) \bar{\eta}_{\infty}(b+p)\right] \\
& \leq \exp \left(\alpha^{*}\left[\sum_{p=1}^{h} \bar{\eta}_{\infty}(b+p)\right]\right),
\end{aligned}
$$

where $\alpha^{*}=\alpha e^{\alpha}$. Looking back to (3.15), we conclude

$$
\begin{aligned}
& \mathbf{P}\left[\sum_{p=1}^{h} \eta_{t}(b+p)>\mathscr{T}(h, v, b)\right] \\
& \quad \leq \exp \left(-\alpha^{*} \frac{1}{2} \frac{v}{N} h^{2}\right) \exp \left[\left(\alpha-\alpha^{*}\right) \mathscr{T}(h, v, b)\right] .
\end{aligned}
$$


The desired result now follows easily: we replace $b$ by $N$ on the right-hand side of (3.25) and segue this bound into (3.17) with an extra, healthy factor of $N$ to account for the sum over $b$. By choosing $\alpha$ small enough, it is not hard to see that we arrive at (3.14).

Let us now marshal all of the facts at our disposal: with probability 1 , with the possible exception of only finitely many $r$ 's, at time $t=N_{r}^{2} q$, there are no more than $N_{r} H \sqrt{\varepsilon}(1-2 v)+v \sqrt{\varepsilon} N_{r} B_{r} H+v \varepsilon N_{r} H^{2}$ particles lying in the region $b_{r} \leq k \leq b_{r}+H \sqrt{\varepsilon} N_{r}$. Further (again, with probability 1, with only finitely many exceptions), in the time interval between $t=N_{r}^{2} q$ and $t=$ $N_{r}^{2}(q+\varepsilon)$, the total amount of leftward displacement that takes place in the entire system is no more than $2 \varepsilon N_{r}^{2}$.

An elementary coupling argument tells us that, regardless of what is to happen in the time interval between $N_{r}^{2} q$ and $N_{r}^{2}(q+\varepsilon)$, the worst case scenario particle configuration at time $t=N_{r}^{2} q$ consistent with the above information is that all of these $N_{r}\left[\sqrt{\varepsilon} H-\left(v\left(2-B_{r}\right)\right) H \sqrt{\varepsilon}+v \varepsilon H^{2}\right]$ particles that are supposed to be in the region $b_{r}<k \leq b_{r}+H \sqrt{\varepsilon} N_{r}$ are as close as possible to the wall at $b_{r}$ and all other particles in the system are lined up, at unit density, in the region $k>b_{r}+H \sqrt{\varepsilon} N_{r}$. Under these circumstances, the most efficient use of the available displacement is to always advance the leading particle. Allowing, as a bound, all of the nearby particles to be consumed in the first instant of time, each subsequent addition to the boundary must now cross a gap of size $N_{r}\left(v\left(2-B_{r}\right) H \sqrt{\varepsilon}-v \varepsilon H^{2}\right)$. Thus, for the total time span of $\varepsilon N_{[r]}^{2}$, we have

$$
\begin{aligned}
B_{r}(q+\varepsilon)-B_{r}(q) \leq & \sqrt{\varepsilon} H N_{r}-\left(v\left(2-B_{r}\right)\right) H \sqrt{\varepsilon}-v \varepsilon H^{2} \\
& +\frac{\sqrt{\varepsilon}}{\left(v\left(2-B_{r}\right)\right) H-v \sqrt{\varepsilon} H^{2}} .
\end{aligned}
$$

We still have the choice of $H$ at or disposal-provided that we choose from a countable set. If we choose the $H$ that optimizes the right-hand side of (3.26), we arrive at a bound of the form stated in (3.12).

REMARK. As discussed before, the preceding estimates easily translate into a proof of Hölder continuity with index $\frac{1}{2}$. Had we been able to do even the slightest amount better, most of the Appendix would have been unnecessary: Hölder continuity with index $\frac{1}{2}$ for the boundaries is just the dividing line between classical and nonclassical behavior for solutions of the heat equation. However, after a moment's reflection on the above proof, it is seen that a better continuity result would have required the knowledge, in the context of the particle system, that the density goes to 0 at the boundary. A direct proof of this fact (which, in light of the continuum results proved in the Appendix, indeed turns out to be the case) has to this date proved elusive. However, it is not difficult to see, in hindsight, that there is a genuine connection between the vanishing of the particle density on the one hand and classical behavior on the other. What is surprising to the authors (again in 
hindsight) is that, in the context of this problem, this issue was settled in the continuum rather than in the particle system.

We are now finally in a position where we can get to the system described in (3.3).

Corollary. Let $T<t^{\star}$ and let $(a(x, t), n(x, t))$ denote any solution to (3.2) on $[-1,+1] \times[0, T]$ that has emerged as a weak subsequential limit of a sequence $\left(a_{r}(x, t), n_{r}(x, t)\right)$ coming from the particle system. Then, with probability 1 , there is a continuous, monotone function, $B(t)$, that is the Stefan boundary for these densities. In particular, $a(x, t)=1$ and $n(x, t)=0$ (a.e.) if $x \leq B(t)$ and $a(x, t)=n(x, t)$ (a.e.) if $B(t) \leq x \leq 1$.

Proof. Let $a(x, t)$ and $n(x, t)$ denote any weak subsequential limits of the sequences $a_{r}(x, t)$ and $n_{r}(x, t)$ that have been taken along the same subsequence, here denoted by $\left(r_{\bar{k}}\right)$. Let $B(t)$ denote the boundary that is produced from a further subsequence, $\left(r_{k}\right)$, as described in Proposition 3.3. Let us prove, for example, that $a(x, t) \equiv 1$ in the region $\{x, t \in[0,2] \times$ $[0, T] \mid x \leq B(t)\}$. It is sufficient to show that if $V$ is any closed set in this region, then

$$
\int_{V} a(x, t) d x d t=|V|,
$$

where $|-|$ denotes ordinary Lebesgue measure. Let $\chi_{V}$ denote the characteristic function (indicator) for the set $V$. Clearly,

$$
\int_{V} a(x, t) d x d t=\lim _{k \rightarrow \infty} \int a_{r_{k}}(x, t) \chi_{V} d x d t .
$$

Using the monotonicity of the $B_{r_{k}}$ 's and the fact that, with probability 1 for any finite (rational) collection $q_{1}, \ldots, q_{m}$ in $[0, T]$, (3.12) holds with $B$ replaced by $B_{r_{k}}$ (except perhaps for finitely many values of $r$ ), it is not hard to show that $B_{r_{k}}(t) \rightarrow B(t)$ uniformly on $[0, T]$. This implies that, with probability 1 , there is some number $\tilde{R}$ such that, for all $r_{k} \geq \tilde{R}$,

$$
V \subset\left\{(x, t) \in[0,2] \times[0, T] \mid x \leq B_{r_{k}}(t)\right\} .
$$

But in the region $\left\{(x, t) \in[0,2] \times[0, T] \mid x \leq B_{r_{k}}(t)\right\}$, we know that $\eta_{r_{k}}=-1$ and thus (3.27) follows. Similar reasoning can be used to demonstrate the second property.

Thus, as far as the particle system is concerned, we may now express our densities in the language of (3.2) or (3.3) as we please. The relevant result from the Appendix will be stated below for convenience:

THEOREM A.3. Consider the system in (3.2) with the initial conditions $B(0)=-1, \rho_{0}(x)$ and boundary value $\rho_{F}(t)$. It is assumed that both $\rho_{0}(x)$ and $\rho_{F}(t)$ are piecewise smooth, bounded above by 1 and below by 0 , and that 
$w(1+x) \geq \rho_{0}(x)$ for some finite constant $w$ and that $\rho_{0}(x) \leq 1+v(x-1)$ for some positive constant $v$. Then there is exactly one solution to this problem and, furthermore, this solution is classical.

REMARK. The classical nature of the solution has no bearing on the current situation, but it is worth noting that on $\left(0, t^{\star}\right)$ the boundary is at least $C^{1}$. As such, away from the endpoints, gradients of the density exist and are continuous at the boundary. Thus, we may actually revert to (1.5), (1.6), (1.7) and (1.8b) to describe this problem.

The proof of our principal result is now almost immediate.

THEOREM 3.4. Consider the interacting particle system on $\mathbb{L}_{N_{r}}$ (where $N_{r}=2^{r} N_{0}$ ) that was described in Proposition 3.1 with boundary and initial conditions that are consistent with those in the statement of Theorem A.3. Then, with probability $1,\left(a_{r}(x, t), n_{r}(x, t)\right)$ converges weakly, in $L^{2}$, to the unique (classical) solution to (3.3).

Proof. Let $(a(x, t), n(x, t))$ denote any limiting density as described in Proposition 3.1. Passing to a further subsequence, if necessary, we see that $(a(x, t), n(x, t))$ enjoys a continuous Stefan boundary. Hence, the limiting particle density actually satisfies (3.3) which, by Theorem A.3, has a unique solution. This is the desired result.

4. An application to two-dimensional interfacial dynamics. Aside from the standard, classical interpretation of the problems treated in the preceding sections, the one-dimensional exclusion process has a well-known application to the study of two-dimensional interfaces. The key observation, due to Rost, is that if 0's are identified as horizontal edges and 1's as vertical edges of an "interface" on $\mathbb{Z}^{2}$, exclusion dynamics among the 0 's and 1's represents a dynamic evolution of this interface. In systems that are a priori infinite, these models were analyzed some time ago: the problem of an infinite corner with complete bias was treated in [12] and the general problem of an infinite corner was solved in [11], Chapter 8.

Of course, there is no reason that one cannot solve, in the sense of hydrodynamic limits, these problems on finite lattices. For example, the exclusion process on $\mathbb{L}_{N}$ with fixed density boundary conditions at the endpoints represents a surface that is constrained to have fixed slopes at its ends. Somewhat less realistically (since the interface has to be a monotone function), one may consider this problem with periodic boundary conditions.

It turns out that these problems all represent the $T \rightarrow 0$ limit of the usual (Gibbs sampler) Glauber dynamics of the stochastic Ising model. Here, the interface is the boundary separating regions of opposite spin type. The above problem with periodic boundary conditions as well as more complicated interfaces on a cylinder (still a function, but not necessarily monotone) was discussed in [13] in this context. In particular, the diffusive behavior that is inevitable in the particle system translates into the motion by (modified) 
mean curvature. This dynamical phenomenon was predicted in [8] and leads to the well-known Lifshitz law. Of considerable interest, then, is the behavior of droplets under this sort of dynamics. In these cases, the generalization of Lemma 3.2 goes through (with some restrictions on the initial shape) and amounts to the statement that the volume of the droplet decreases linearly with time [2]. This is a weak form of the Lifshitz law.

In general, such problems appear to be difficult to model as particle systems. However, it is our understanding that the methods in [13] can easily be extended to cover these cases, a result that is due to Spohn. Notwithstanding, the behavior of droplets near the edge of a system is still of some interest.

Suppose in the region $\left\{i \in \mathbb{Z}^{2} \mid i_{1} \geq 0\right\}$ there is an Ising ferromagnet with the usual nearest-neighbor interactions. If $I_{t}$ is the interface at time $t$, the Gibbs sampler Glauber dynamics at zero temperature dictates that if $J$ is an interface that can be obtained from $I_{t}$ by the flip of a single spin, $I_{t} \rightarrow J$ at rate 1 if the total length of the interface is preserved and at rate 2 if the total length of the interface decreases. All other moves are suppressed. If the interface extends into the wall at $i_{1}=0$, it is easily seen that the interfacial transitions in this vicinity are irreversible. In particular, if the interface is a monotone (say nonincreasing) function, the behavior at this end is modeled by just the particle system that was described in Section 3 [i.e., (1.4); the enhancement of the transition rate at the boundary turns out to be of no consequence]. In these systems, the behavior of finite droplets is of considerably greater interest than the behavior in a semiinfinite system. Unfortunately, the boundary conditions we have used at the "non-Stefan" end of the system are not particularly realistic for this sort of application.

For the problem of an Ising droplet in the corner of the sample (now defined as the positive quadrant), a particle-systems approach along the lines we have been discussing is applicable. However, the dynamics must now exhibit complete particle-hole symmetry; in particular, we must extend the single-particle space to include an additional state that can best be described as a frozen hole. The dynamics between particles, holes and frozen particles is exactly as described in (1.3) and (1.4), while the dynamics between particles, holes and frozen holes is identical after a switching of labels. However, in this extended system, a major casualty is notation: assigning numbers to the states $\eta_{t}(k)$ here is not particularly efficient. Since we will end up describing this problem using the enthalpy and auxiliary density, we may as well use this notation from the outset. We thus have

$$
\eta_{t}(k)=\left(\begin{array}{l}
a_{t}(k) \\
n_{t}(k)
\end{array}\right)
$$

with $a_{t}$ and $n_{t}$ taking on the values 0 or 1 . The process is defined by the action of the generator $\mathscr{L}_{\mathbb{L}_{N}}$ on functions $f(-)$ of the configurations on $\mathbb{L}_{N}$ :

$$
\mathscr{L}_{\mathbb{L}_{N}} f(\eta)=\frac{1}{2} \sum_{\substack{i \in \mathbb{L}_{N} \\ j=1 \pm i}}\left(\left[f\left(\eta_{D}^{[i, j]}\right)-f(\eta)\right]+\kappa\left[f\left(\eta_{F}^{[i, j]}\right)-f(\eta)\right]\right)
$$


where, first, the switched configurations always satisfy $\eta_{D}^{[i, j]}=\eta=\eta_{F}^{[i, j]}$ unless $a(i)=n(i)$ and $a(j)=n(j)$. Further, the "diffusive" switches in (4.2) are limited by $\eta_{D}^{[i, j]}=\eta$ unless $a(i \pm 1)=n(i \pm 1)$ and $a(j \pm 1)=n(j \pm 1)$ and otherwise they are defined by the usual

$$
\eta_{D}^{[i, j]}(k)= \begin{cases}\eta(k), & \text { if } k \neq i, k \neq j \\ \eta(j), & \text { if } k=i \\ \eta(i), & \text { if } k=j .\end{cases}
$$

Next, the "freezing" switches are defined by

$$
\begin{aligned}
& \eta_{F}^{[i, j]}(k)=\eta(k) \quad \text { if } k \neq i, k \neq j \\
& \eta_{F}^{[i, j]}(i)=\left(\begin{array}{l}
1 \\
0
\end{array}\right) \text { and } \eta_{F}^{[i, j]}(j)=\left(\begin{array}{l}
0 \\
0
\end{array}\right)
\end{aligned}
$$

if

$$
\begin{array}{r}
\left.a(i)=0, a(j)=1 \text { and } \begin{array}{r}
a(i+1) \\
n(i+1)
\end{array}\right) \text { or }\left(\begin{array}{l}
a(i-1) \\
n(i-1)
\end{array}\right)=\left(\begin{array}{l}
1 \\
0
\end{array}\right) \\
\text { while } a(j \pm 1)=n(j \pm 1)
\end{array}
$$

and similarly with the roles of $i$ and $j$ reversed, and

$$
\eta_{F}^{[i, j]}(i)=\left(\begin{array}{l}
0 \\
1
\end{array}\right) \quad \text { and } \quad \eta_{F}^{[i, j]}(j)=\left(\begin{array}{l}
1 \\
1
\end{array}\right)
$$

if

$$
\begin{array}{r}
a(i)=1, a(j)=0 \quad \text { and }\left(\begin{array}{l}
a(i+1) \\
n(i+1)
\end{array}\right) \text { or }\left(\begin{array}{l}
a(i-1) \\
n(i-1)
\end{array}\right)=\left(\begin{array}{l}
0 \\
1
\end{array}\right) \\
\text { while } a(j \pm 1)=n(j \pm 1)
\end{array}
$$

and similarly with the roles of $i$ and $j$ reversed. In (4.2) we have tacitly included formal terms involving $\eta(N+1)$ and $\eta(-[N+1])$; these are assumed to take the values $\eta(N+1)=\left(\begin{array}{l}0 \\ 1\end{array}\right)$ and $\eta(-[N+1])=\left(\begin{array}{l}1 \\ 0\end{array}\right)$, respectively; elsewhere in the initial condition we will be assuming that $a_{0}(k)=$ $n_{0}(k)$. We have also not defined switches for the situations where both the sites $i$ and $j$ have frozen neighbors. In our particular instance, only one such transition occurs, and this is the move that signals the end of the simulation.

The continuum description of the purported limit is, classically,

$$
\frac{\partial n}{\partial t}=\frac{\partial^{2} n}{\partial x^{2}}, \quad B(t) \leq x \leq C(t),
$$


with some initial condition $n(x, 0)$, the boundary condition at the moving boundaries given by $n(B)=0$ and $n(C)=1$ and the Stefan conditions

$$
\begin{aligned}
& \frac{d B}{d t}=\nabla n(B(t), t), \\
& \frac{d C}{d t}=-\nabla n(C(t), t) .
\end{aligned}
$$

In the weakest form, the Stefan equation is exactly (3.2) with the boundary term absent (and no boundary restriction on the test function). Therefore, the intermediate version is

$$
\begin{aligned}
\int_{B(t)}^{C(t)} n(x, t) G(x) d x-\int_{B(0)}^{C(0)} n(x, 0) G(x) d x \\
=\int_{0}^{t} \int_{B(s)}^{C(s)} n(x, s) \frac{\partial^{2} G}{\partial x^{2}} d x d s+\int_{0}^{t} \int_{C(s)}^{\infty}\left(\frac{\partial^{2} G}{\partial x^{2}}\right) d x d s \\
\quad-\int_{B(0)}^{B(t)} G(x) d x,
\end{aligned}
$$

where $G$ is any time-independent test function of compact support.

Unfortunately, far less is known about the solutions to the above system than in the case with a single boundary. In particular, we can only show uniqueness under the assumption of complete symmetry in the initial condition: $\rho_{0}(x)=1-\rho_{0}(-x)$. However, this covers a case of principal concern, namely that of a droplet that is initially square. Henceforth, we will restrict attention to this case. Our primary result of this section will be proved along lines that follow very closely the previous derivations.

THEOREM 4.1. Consider the interacting particle system defined on $\mathbb{L}_{N_{r}}$ described in (4.1) to (4.4) with $0<\kappa<\infty$ and initial conditions corresponding to $B(0)=-1, C(0)=+1$ with $\rho(x, 0)=1$ if $x>0$ and $\rho(x, 0)=0$ if $x<0$. Let $\left(a_{r}(x, t), n_{r}(x, t)\right)$ denote the quantities defined naturally from $\eta(x, t)$ as in (2.8). Then, with probability $1,\left(a_{r}(x, t), n_{r}(x, t)\right)$ converges weakly, in $L^{2}$, to the unique solution with this initial condition.

Proof (Sketch). In this system, we define $b_{r}(t)$ exactly as in (3.4)-with the obvious modification for this section's notation-and, similarly,

$$
c_{r}(t)=N_{r}+\sum_{k \leq N_{r}} \delta_{\left(\eta_{t}(k),\left(\begin{array}{l}
0 \\
1
\end{array}\right)\right.} .
$$

Thus, explicitly, the $C$-boundary is the location of the particle that is right on the edge of the line of frozen 0's. The first step is to derive the discrete analog of (3.2). Following the steps of Proposition 2.1, we get this equation, in martingale form for the quantities $M_{r}(G)$ defined exactly as in (2.15)-(2.17) 
(without the $R_{F}$ terms and with the new meanings assigned to the $n_{r}$ 's and $a_{r}$ 's), except that this time, the unwanted leftover terms take the form

$$
\begin{aligned}
& (\kappa-1)\left[g_{t}\left(b_{r}\right)-g_{t}\left(b_{r}+1\right)\right] a_{t}\left(b_{r}+1\right) \\
& \quad+(\kappa-1)\left[g_{t}\left(c_{r}-1\right)-g_{t}\left(c_{r}\right)\right]\left[1-a_{t}\left(c_{r}-1\right)\right] .
\end{aligned}
$$

However, these terms are dispensed with by using exactly the argument found in the remark following Proposition 2.1. Evidently, the averages contain the desired weakly converging subsequences. A quadratic variation calculation along the lines of Proposition 2.2 can be carried out. After some effort, it can be checked that the same sorts of terms with the expected additional leftovers caused by the presence of the extra boundary emerge along with the desired result, namely that, with probability 1 , random subsequences converge to weak solutions of the Stefan equation with two boundaries.

Next, the arguments of Proposition 3.3 can be applied with only a few modifications. First, it turns out that $\mathscr{Q}_{F}(t)$ is replaced by $\frac{1}{2} t$ in all matters that relate to the displacement. Second, the argument must officially be performed for each boundary individually (although the separate arguments are identical). Third, focusing attention on the left half of the configuration, $\{-N,-N+1, \ldots, 0\}$ [where, with probability 1 , with only finitely many exceptions, the boundary $B_{r}(t)$ is located], the configurations here may be dominated by a setup that has unit density at the origin and zero density on the left end.

Finally, for the case of this particular initial distribution, the results of Theorem A.3 can be extended to the two-boundary problem (Proposition A.5), and, putting these ingredients together (as was done for the single-boundary case), the desired result is established.

REMARK. From the limiting solution, $(B(t), C(t), n(x, t))$, the surface can be reconstructed, parametrically, by the formulas

$$
\begin{aligned}
& Y(s, t)=-B(t)-Q(s, t), \\
& X(s, t)=s-B(t)-Q(s, t),
\end{aligned}
$$

where $Q(s, t)=\int_{B(t)}^{s} \rho(x, t) d x$. This shape is achieved by the stochastic model, for example, in the sense that, for a.e. $t$ in $[0,1),\left(X_{r}(s, t), Y_{r}(s, t)\right) \rightarrow$ $(X(s, t), Y(s, t))$. More refined notions of convergence in these problems will be pursued in the future.

\section{APPENDIX}

We will now proceed with the analysis of the Stefan problem that is defined, classically, by (1.5), (1.6), (1.7) and (1.8b) or, in more generality, by (3.3). In order to simplify the forthcoming discussion, we will analyze this problem on $[0,1] \times\left(0, t^{\star}\right)$ (where $t^{\star}$ is defined below). Most of what is to follow will be the analysis of a reasonably general single (moving) boundary problem subject to the Stefan condition. We are looking for some $B(t)$ that is 
continuous, monotone and has $B(0)=0$ and $B\left(t^{\star}\right)=1$. Then we want some $\rho=\rho_{B}$ that satisfies the heat equation in $B(t) \leq x \leq 1$, vanishes at $B(t)$, has some initial condition and equals some prescribed function at $x=1$. Furthermore, the pair $B$ and $\rho_{B}$ have to satisfy "the Stefan condition" that the speed of the boundary is given by the flux of $\rho_{B}$ through the boundary. In order to keep things simple, we will assume that $\rho_{B}(x, 0)$ is piecewise continuous. We make the further technical assumptions that $w x<\rho_{0}(x)<1-v(1-x)$ for some $v>0$ and $w<\infty$. At the fixed boundary, $x=1$, we have $\rho$ equal to $\rho_{F}(t)$, also assumed to be piecewise continuous. We define $\mathscr{Q}_{F}(t)$ to be the integral of $\rho_{F}, \Delta_{0}$ to be the integral of $(1-x) \rho_{0}(x)$ and

$$
t^{\star}=\inf \left\{t \mid \mathscr{Q}_{F}(t)+\Delta_{0}\right\}=\frac{1}{2} .
$$

If the given $\rho_{F}(t)$ is not defined on a large enough interval to have a $t^{\star}$, it can be extended, continuously, until this is the case. We will assume, without loss of generality, that this extension has been performed.

Under these circumstances, we will show that there is a unique solution to the Stefan problem. Furthermore, we show that this solution is "smooth"; that is, $B(t)$ is Hölder continuous (with index 1). With this in mind, consider the problem on $[-1,+1]$ with two boundaries. Given the existence of a full solution, the center line acts as a boundary condition for the left and right halves. Thus, according to the claims made above, it follows that the Stefan boundaries will be smooth. It turns out that if the full problem has two smooth boundaries and the initial condition is symmetric (with respect to reflections across the midline and the exchange of $\rho$ with $1-\rho$ ), it is not particularly difficult to show that the resulting solution must be symmetric and is unique.

Certain aspects of this appendix have appeared elsewhere, for example, [4] and [3] and undoubtedly some other places as well. In [4], the single-boundary problem was investigated. Under rather similar hypotheses, it was shown that a solution with a Hölder continuous boundary exists. Unfortunately for us, uniqueness was only established among the class of all possible solutions with Hölder continuous boundaries. Hence, even for the single-boundary problems, the result is not quite sufficient for the analysis of an interacting particle system. In [3], substantial progress was made using only the weakest form of the Stefan equation - the analog of (3.2) — without any explicit mention of the boundaries. However, in [3] the flux rather than the density was specified at the fixed boundary: a procedure that is difficult to implement in an interacting particle system. Furthermore, a number of technical assumptions were made in [3] concerning the initial and the boundary conditions that are either violated or unverifiable in the systems we consider here.

Starting with (3.3) and using $G(x)=(1-x)$-which turns out to be the only equation explicitly involving $B$ that is needed-we have

$$
B(t)-\frac{1}{2} B(t)^{2}+\int_{B}^{1}[1-x] \rho_{B}(x, t) d x=\Delta_{0}+\mathscr{Q}_{F}(t) .
$$

Let us first observe that the combination of (A.2) forces $B(t)$ to 1 as $t \rightarrow 1$. 
Proposition A.1. Let $\left(B(t), \rho_{B}\right)$ be a solution to the Stefan problem as described that is defined for all $t$ in $\left[0, t^{\star}\right)$. Then $\lim _{t \rightarrow t^{\star}} B(t)=1$.

Proof. Since $\rho_{B}$ and $\rho_{F}$ do not go negative, we have

$$
B(t)-\frac{1}{2} B^{2}(t) \leq \mathscr{Q}_{F}+\Delta_{0} .
$$

Hence, by the definition of $t^{\star}$, we see that for $t<t^{\star}, B(t)$ does not reach 1 . As for the opposite bound, since $\rho_{F} \leq 1$ and the initial density is bounded above by $1-v(1-x)$, by an argument similar in spirit to the one used in the particle system in Proposition 3.3, it can be shown that for any $x$ in $[B(t), 1]$, $\rho(x, t) \leq 1+v(1-x)$; thus, for $t<t^{\star}$, we obtain

$$
\frac{1}{2}-\mathscr{Q}_{F}+\Delta_{0} \geq \frac{v}{3}(1-B)^{3},
$$

so that, indeed, $B(t) \rightarrow 1$.

REMARK. With a bit more work one can replace (A.4) with a bound more along the lines of (A.3). Thus, one has, as $t \rightarrow t^{\star}$, the asymptotic behavior $B(t) \sim 1-$ const. $\left[\frac{1}{2}-\left(\mathscr{Q}_{F}+\Delta_{0}\right)\right]^{1 / 2}$.

For a general problem with a particular moving boundary given by $D(t)$ and a $\rho_{D}$ satisfying the heat equation in the region $D(t) \leq x \leq 1$, vanishing at $D(t)$ and otherwise satisfying the same initial and boundary conditions as the above Stefan problem, the analog of (A.2) reads

$$
\Phi_{D}(t)-\int_{0}^{t} D \dot{\Phi}_{D} d s+\int_{D}^{1}[1-x] \rho_{D}(x, t) d x=\mathscr{Q}_{F}(t)+\Delta_{0},
$$

where $\Phi_{D}(t)$ is the total outward flux through $D$ at time $t$. In the above, we have tacitly assumed that all terms are well defined. Under our working conditions, that is, $\rho$ bounded and $D$ bounded away from the line $x=1$ and (as will follow shortly) Hölder continuity for $D(t)$ with index exceeding $\frac{1}{2}$, it is known that the solution behaves completely classically (cf. [1], page 247, Theorem 14.41). In particular, $\rho_{D}$ possesses a continuous derivative at the boundaries for any $t$ in $(0,1)$. Hence, we may use

$$
\dot{\Phi}_{D}=\nabla_{\rho_{D}}(D(t), t)
$$

as our definition of the outward flux.

The outline of our program is as follows. Let us start things off with some reasonable boundary $D(t)$ that is either too large or too small to be the Stefan boundary with the stated initial condition at $t=0$ and the boundary condition at $x=1$. If $D$ were "too small," this would be signaled by the fact that $\Phi_{D}>D$. An improved attempt at a solution would then be to use $\Phi_{D}$ to create a better approximation. This procedure can be cycled through repeatedly. The obvious choice for an initiating boundary is $D \equiv 0$ and, without thinking too hard, it is clear that the succession of boundaries increases.

As for the other side, we might try the same sort of approach; however there are a few small difficulties that must be surmounted. One of these is a 
reasonable choice of the first boundary. We will discuss this matter in a bit of detail below. The second difficulty is that with an upper sequence of this form, it is inevitable that the flux will actually catch up with the boundary at some time $t \leq t^{\star}$. To see this, suppose that $D$ is "too large" to be a Stefan boundary. Although this, vaguely, means that $D$ is larger than any "true" Stefan boundary, we had better see $\Phi_{D}<D$ - at least at first-or we would know immediately that we were doing something wrong. However, if $D \rightarrow 1$ (which is the only sensible thing to do) and $\Phi_{D}>\Phi_{B}=B$, it is inevitable that a collision will occur. The cure for this problem, which is remarkably simple in hindsight, is to define the new boundary to be the flux through the old one, until the flux catches up, whereafter we simply set the new boundary equal to the old one. (In fact, for a "bad" choice of a startup boundary, the same sort of thing might happen in the lower sequence, namely that the boundary could catch up with the flux. If so, this "problem" could be cured in pretty much the same fashion as with the upper sequence.) As a result of these procedures, we get two sequences, denoted by $\left(L_{k}, \rho_{L_{k}}\right)$ and $\left(U_{k}, \rho_{U_{k}}\right)$, of approximate solutions to the Stefan problem. It is not hard to believe (or anticipate) that these objects converge to genuine solutions of the Stefan problem. However, it will also turn out that the limiting boundaries, denoted by $L$ and $U$, are smooth and that if $B$ is the boundary corresponding to any other solution, $L \leq B \leq U$. Finally (and here we could borrow directly from [4]), we will show that $L=U$, thus establishing uniqueness.

In the course of our analysis, we will have use for an auxiliary function that we will denote by $A(t)$. Among other things, $A(t)$ will serve as the startup function for the upper sequence that was alluded to above. Under the easiest circumstances, namely $\rho_{0}(x) \equiv 0$ and $\rho_{F}(t)$ a constant (or bounded away from 0 ), the motivated choice of $A(t)$ is the solution to the equation $A-\frac{1}{2} A^{2}=\mathscr{Q}_{F}(t)$. This would provide us with four properties (listed below) that are useful in our analysis. On a preliminary reading, the preceding can be thought of as the $A(t)$ under the above-mentioned restricted circumstances.

Definition. Let $T<t^{\star}$ and let $A(t)$ denote any particular function that satisfies the following four conditions:

(i) $A(t)$ is piecewise smooth (differentiable) on $[0, T]$.

(ii) $A(t) \rightarrow 0$ as $t \rightarrow 0$ and $A(t) \leq 1$ on $[0, T]$.

(iii) There is some $\varepsilon>0$ such that for all $t \in[0, T], \dot{A(t)} \geq \varepsilon /(1-A(t))$.

(iv) If $B(t)$ is the boundary in a solution to the Stefan equation under consideration, then $A(t) \geq B(t)$.

REMARK. Under our working conditions, it is not so difficult to construct such an $A(t)$. First, let us define $\mathscr{Q}_{F}^{*}(t)=\mathscr{Q}_{F}(t)$ if $\rho_{F}$ is bounded away from 0 , and otherwise take all the mass in $\left[T, t^{\star}\right]$ and redistribute it evenly on $[0, T]$ :

$$
\mathscr{Q}_{F}^{*}(t)=\mathscr{Q}_{F}(t)+\frac{t}{T}\left[\mathscr{Q}_{F}\left(t^{\star}\right)-\mathscr{Q}_{F}(T)\right] .
$$


Thus, we have $\mathscr{Q}_{F}^{*}(t) \geq \mathscr{Q}_{F}(t)$ and also that $\dot{\mathscr{Q}}_{F}^{\star} \geq \varepsilon_{1}$ for some $\varepsilon_{1}>0$ on $[0, T]$. Now consider $A_{1}(t)$ defined by

$$
A_{1}(t)-\frac{1}{2} A_{1}(t)^{2}=\Delta_{0}+\mathscr{Q}_{F}^{\star} .
$$

Clearly, $A_{1}(t)$ satisfies everything except the first part of condition (ii). Next, by using the fact that $\rho_{0}(x) \leq \max \{w x, 1\}$ (which also serves as a bound for $\rho$ at positive times) and an explicit trial function that depends on $B(t)$ [e.g., $G(x) \approx \max \{0,1-x / B(t)\})$, it is easy to show that $B$ is bounded above by $c w t$, where $c$ is a constant larger than 1 but of the order of unity. It is clear that cwt satisfies all of the conditions except the second part of condition (ii); we may define $A(t)$ to be the minimum of cwt or $A_{1}(t)$.

We also remark that the $A(t)$ as defined (possibly) has some dependence on $T$ but this will not be reflected in our notation.

In order to proceed further, we will need a technical lemma to ensure that each stage of the process is well defined. In addition, and of equal importance in the overall scheme of things, this lemma provides us with a uniform estimate for the Hölder continuity of all the boundaries involved in the approximating sequences.

LEMma A.2. Let $T<t^{\star}$ and consider the heat equation with the identical initial condition at $t=0$ and boundary condition at $x=1$ as that under consideration but with a moving boundary $D(t)$ as discussed in (A.5) and (A.6). Suppose that $D$ is differentiable for times $t \leq T$. Let $A(t)$ denote the function discussed above and further suppose that $D(t) \leq A(t)$. Then there is a constant $W \equiv W(T)<\infty$ such that, if

$$
\dot{D} \leq \frac{W}{1-A(t)},
$$

then, for all $t \in[0, T]$,

$$
\dot{\Phi}_{D} \leq \frac{W}{1-A(t)} .
$$

PROOF. Our proof will be to produce a trial function that "dominates" the actual density. Consider $x$ in the region between $D(t)$ and the distance that $A(t)$ keeps from the wall: $D(t) \leq x \leq 1-A(t)$. For convenience, let $\vartheta$ denote the normalized variable

$$
\vartheta=\frac{x-D}{1-A} .
$$

We will express our trial function in terms of the variable $\vartheta$. Let $\psi_{W}(\vartheta)$ denote the function

$$
\psi_{W}(\vartheta)=\int_{0}^{W \vartheta} \exp (-s) \exp \left((1 / 2 W) \varepsilon s^{2}\right) d s,
$$


where $\varepsilon \equiv \varepsilon(T)$ is the parameter in the function $A(t)$. We claim that for all $W$ sufficiently large, $\psi_{W}(1) \geq 1$. Indeed, for $W \gg 1, \psi_{W}(1)>\left[1-e^{-W / 2}\right]+$ $e^{(1 / 8) \varepsilon W}\left[e^{-W / 2}-e^{-W}\right]$, which clearly exceeds 1 if $W$ is sufficiently large. Similarly, for any fixed $\vartheta>0, \psi_{W}(\vartheta) \rightarrow 1$ as $W \rightarrow \infty$.

Denoting by $\psi$ the function that is $\psi_{W}(\vartheta)$ for $\vartheta \leq 1$ and $\psi_{W}(1)$ beyond $\vartheta=1$, we see that, in the region $\vartheta \leq 1$,

$$
\frac{\partial \psi}{\partial t} \geq \frac{1}{(1-A)^{2}}[-W+\varepsilon \vartheta]\left[\frac{d \psi}{d \vartheta}\right] \geq \frac{\partial^{2} \psi}{\partial x^{2}},
$$

while both terms vanish in the region $\vartheta \geq 1$. Evidently, $\partial \psi / \partial t-\partial^{2} \psi / \partial x^{2} \geq 0$, so we may conclude that $\psi$ has enjoyed the benefits of a positive heat source. Furthermore, at $t=0$ (where $\vartheta=x$ ), we may write, for $W x \leqq 1, \psi>1-e^{-W x}$. Thus, clearly, $\psi$ exceeds the initial density if $W$ is large compared with $w$. Finally, $\psi$ satisfies the relevant boundary condition at $x=D$ and is at least as big as the boundary condition prescribed for $x=1$ (with no flux at this boundary). It follows that $\nabla \psi(D(t), t) \geq \nabla \rho_{D}(D(t), t)$. However, the former is seen to be $W /(1-A(t))$.

Our first theorem constitutes a fairly complete statement concerning the so-called one-phase Stefan problem with an inward moving boundary.

THEOREM A.3. Consider the Stefan problem on $[0,1] \times[0, T]$ defined (classically) by a density $\rho_{B}(x, t)$ and a boundary $B(t)$. It is assumed that the boundary is continuous and nondecreasing. The density $\rho_{B}$ satisfies the heat equation for $B(t)<x<1$ for all $t$ in $(0, T]$, vanishes at $x=B(t)$, satisfies the initial condition $\rho_{B}(x, 0)=\rho_{0}(x, 0)$ with the stipulations described prior to (A.1) and the boundary condition $\rho_{B}(1, t)=\rho_{F}(t)$, where $\rho_{F}(t)$ is bounded between 0 and 1 , (piecewise) continuous on [0,T], and integrates to some number $\mathscr{Q}_{F}(T)<\frac{1}{2}-\Delta_{0}$, where $\Delta_{0}$ is also defined just prior to (A.1). Finally, $B(t)$ and $\rho_{B}(x, t)$ are connected by the Stefan condition which may be expressed formally as $\dot{B}=\nabla \rho_{B}(B, t)$ or by (A.2). Then there is exactly one solution to this problem; the resulting $B(t)$ is Hölder continuous (with index 1) on $[0, T]$ and the density $\rho_{B}(x, t)$ is classical.

Proof. Let $\left(B, \rho_{B}\right)$ denote a solution to the Stefan problem on $[0,1]$ and let $T<t^{\star}$. Let us start by restricting attention to the action on $[0, T]$. As mentioned earlier, we will use the notation $D, \rho_{D}$ and $\Phi_{D}$ to denote the boundary, density and outward flux for a problem which (aside from the differences in boundaries) has the same setup as the above-described problem. Consider the sequence of boundaries $\left(L_{k}\right)$ defined by

$$
L_{0} \equiv 0
$$

and

$$
L_{k+1}=\Phi_{L_{k}}
$$


An elementary argument using the maximum principle shows that, for all $k$, $L_{k+1} \geq L_{k}$. Obviously, this is true for $k=0$, so, given that $L_{k+1} \geq L_{k}$, we must demonstrate that $\Phi_{L_{k+1}} \geq \Phi_{L_{k}}$. Now, for finite $k$, all quantities are sufficiently smooth to allow classical reasoning to be applied. On the boundary, $L_{k+1}$, the quantity $\rho_{L_{k+1}}$ vanishes while (unless $L_{k+1}=L_{k}$ ), $\rho_{L_{k}}$ does not. It follows that in the region to the right of $L_{k+1}$, we have $\rho_{L_{k}} \geq \rho_{L_{k+1}}$. However, these quantities are equal at $x=1$ and this implies $\left.\nabla \rho_{L_{k}}\right|_{x=1} \leq$ $\left.\nabla \rho_{L_{k+1}}\right|_{x=1}$. Integrating this inward flux, we obtain $\Phi_{L_{k}}+\int \rho_{L_{k}} \leq \Phi_{L_{k+1}}+$ $\int \rho_{L_{k+1}}$, and (because $\rho_{L_{k}} \geq \rho_{L_{k+1}}$ ) the desired inequality is established.

We claim that the limit is Hölder continuous. Indeed, for $t \leq T$, combining (A.2), (A.5), the fact that $A>B$ and the assumption that $L_{k} \leq B-$ which is certainly true if $k=0$-we get

$$
A-\frac{1}{2} A^{2} \geq B-\frac{1}{2} B^{2} \geq \Phi_{L_{k}}-\int L_{k} \dot{\Phi}_{L_{k}} \geq \Phi_{L_{k}}-\frac{1}{2} \Phi_{L_{k}}^{2},
$$

where we have used the fact that $\rho_{L_{k}} \geq \rho_{B}$. [We state this without proof. For smooth $B$, this is an obvious consequence of the maximum principle. For general $B(t)$, this could require something of an explanation, but since both $B$ and $L_{k}$ are monotone increasing, the result can be readily established from the weak form of the heat equation.] Thus, $A(\geq B) \geq L_{k+1}$ and Lemma A.2 may be applied, repeatedly. Denoting by $L(t) \equiv \lim _{k \rightarrow \infty} L_{k}$, we see that $L(t)$ is Hölder continuous on $[0, T]$ and, further, that $L(t) \leq B(t)$. It is not hard to show that the pair $\left(L, \rho_{L}\right)$ actually satisfy the Stefan equation. First, it is easily argued that $\rho_{L}=\lim _{k \rightarrow \infty} \rho_{L_{k}}$. Indeed, the sequence is monotone and hence, by monotone convergence, satisfies the (weak) heat equation in the region $x>L$. Moreover, using the trial function of Lemma A.2 to bound the density, it is seen that $\rho_{L_{k}}(L(t), t)$ actually converges to 0 . Since the final $L$ has already been established as smooth, the function $\rho_{L}$ must be the unique solution to the heat equation in the above domain. We now write (A.5) in the form

$$
\begin{aligned}
\Phi_{L_{k}} & -\frac{1}{2} \Phi_{L_{k}}^{2}-\int_{0}^{t}\left(L_{k}-\Phi_{L_{k}}\right) \dot{\Phi}_{L_{k}} \\
& +\int_{L_{k}}^{1}(1-x) \rho_{L_{k}}=\mathscr{Q}_{F}(t)+\Delta_{0} .
\end{aligned}
$$

Whence, using the uniform bound of $\dot{\Phi}_{L_{k}}$ and monotone convergence,

$$
L-\frac{1}{2} L^{2}+\int_{L}^{1}(1-x) \rho_{L}=\mathscr{Q}_{F}(t)+\Delta_{0} .
$$

Let us now turn attention to the sequence, $\left(U_{k}\right)$, of upper bounds. As was mentioned earlier, we will define $U_{0}(t)=A(t)$. It is noted that, as $t \downarrow 0$, $\Phi_{U_{0}}<U_{0}$. In particular, we may use the bounds $\rho_{0}(x) \leq w x$ and $K_{1} w t \leq$ $A(t) \leq K_{2} w t$ with $w$ large and $K_{1}$ and $K_{2}$ both larger than 1. Plugging in the upper bounds on $\rho$ and $A$, we get an upper bound on $\Phi$. This problem can be solved asymptotically, and as $t \downarrow 0$ (not surprisingly) the limiting flux is 
that obtained for a stationary wall, namely, $w t$. Things are starting off well; however, there may come a time $t_{0}^{*}<T$ when the flux catches up with the boundary. If this is the case, we define

$$
U_{1}(t)= \begin{cases}\Phi_{U_{0}}(t), & t \leq t_{0}^{*}, \\ U_{0}, & t \geq t_{0}^{*} .\end{cases}
$$

Suppose we have worked the analogous procedure $k$ times and have found, for early times, that $U_{k-1} \geq \Phi_{U_{k-1}}$ (i.e., $t_{k-1}^{*} \geq T$ ). Then it follows from an argument using the maximum principle that $\Phi_{U_{k}} \leq \Phi_{U_{k-1}}$. If the flux catches up at some time $t_{k}^{*}$ before $T$, define

$$
U_{k+1}(t)= \begin{cases}\Phi_{U_{k}}(t), & t \leq t_{k}^{*}, \\ U_{k}, & t \geq t_{k}^{*} .\end{cases}
$$

It is noted, in passing, that the sequence $\left(t_{k}^{*}\right)$ is monotone and, in fact, converges to $t^{\star}$. Thus, on the interval $[0, T]$, the resetting procedure need only be done a finite number of times. Denoting by $U(t)$ the limit of $U_{k}$, it is clear that $U$ is Hölder continuous and, as in the case of the lower sequence, that the resulting pair $\left(U, \rho_{U}\right)$ satisfy the Stefan equation. These facts follow from essentially the same set of arguments that were used in the case of the lower sequence.

We now claim that if $B(t)$ is the boundary associated with any solution to the Stefan problem, then $U(t) \geq B(t)$. Indeed, suppose that $B(t)$ is such a boundary. We know that $U_{0}>B$; let us suppose that $U_{k}>B$. For $t \geq$ $t_{k}^{*}$-assuming that the latter is less than $T$-it is safe to conclude that $U_{k+1}>B$. For $t<t_{k}^{*}$, an examination of (A.2) and (A.5), together with the fact that $\rho_{B}(x, t) \geq \rho_{U_{k}}(x, t)$, gives us

$$
B-\frac{1}{2} B^{2} \leq \Phi_{U_{j}}-\int_{0}^{t} U_{j} \dot{\Phi}_{U_{j}}
$$

By hypothesis, for all these times, $U_{j}>\Phi_{U_{j}}$; putting this into the integral, we may conclude that $U_{k+1}>B$.

Now we are in the position where we have upper and lower solutions, $\left(L, \rho_{L}\right)$ and $\left(U, \rho_{U}\right)$, for times up through any $T<t^{\star}$; if $B$ is any other Stefan boundary associated with this problem, $L(t) \leq B(t) \leq U(t)$ and, provided that we keep $T$ away from $t^{\star}$, both $U$ and $L$ are smooth. Clearly, the theorem is proved if we can show that $U=L$. As a matter of fact, uniqueness - among the class of solutions with Hölder continuous boundaries-was established in [4], so we may borrow this fact to proclaim a full blown uniqueness result. However, the following argument is also of interest.

Observe, as a consequence of Proposition A.1 that, as $t \rightarrow t^{\star}$, both $L$ and $U$ tend to 1. Suppose that there is a $T_{a}<t^{\star}$ such that, for times in $\left[0, T_{a}\right)$, $U>L$. Since these moving boundaries are known to be smooth, we can use 
the (strong) maximum principle at the right boundary to demonstrate that, for certain times, $\nabla \rho_{U}(1, t)>\nabla \rho_{L}(1, t)$. However,

$$
\int_{0}^{t} \nabla \rho_{U}(1, s) d s=U(t)+\int_{U}^{1} \rho_{U}(x, t) d x-\int_{0}^{1} \rho_{0}(x) d x
$$

and similarly for $L$. Hence, we would conclude that, for times $t>T_{a}$, there is some positive $s$ for which

$$
U(t)+\int_{U}^{1} \rho_{U}(x, t) d x \geq L(t)+\int_{L}^{1} \rho_{L}(x, t) d x+\varsigma
$$

and thus $\lim _{t \rightarrow t^{\star}} U(t)>1$, an impossibility.

Now that we know the solution has a Hölder continuous boundary, it follows that the solution inside is classical. From this, further continuity properties of the boundary can be obtained-presumably all the way up to $C^{\infty}$. We will not pause for a full pursuit of this topic (such things have appeared in the literature); we will be content with the following.

Corollary. For any $T<t^{\star}$, the boundary $B(t)$ corresponding to the solution of the Stefan problem described in the statement of Theorem A.3 is of class $C^{1}(0, T]$.

Proof. If the density is classical, we have $\dot{B}(t)=\nabla \rho(B(t), t)$. Since the right-hand side of this relation is well defined, $B(t)$ is evidently of class $C^{1}$ on $(0, T]$.

We now turn to the two-boundary problem defined, classically, in (4.5) and (4.6) or, more generally, in (4.7). To maintain continuity with the preceding derivation, we will take $x$ to lie between 0 and 2 (with $0 \leq t<1$ ). Thus, our initial condition now reads $\rho_{0}(x)=0$ if $x<1$ and $\rho_{0}(x)=1$ if $x>1$. As a serviceable version of the Stefan equation, we may use (with apologies for unfortunate notation)

$$
2 B-\frac{1}{2} B^{2}+\int_{B}^{C}(2-x) \rho_{B C}=t+\frac{1}{2},
$$

where $\rho_{B C}$ is defined-and satisfies the heat equation-in $B(t) \leq x \leq C(t)$. A straightforward check will reveal that this is just an example of (4.7) along with the stipulation of mass conservation which, for this system, reads $\int_{B}^{C} \rho_{B C} d x=1-B$. We now demonstrate that (A.19) (along with the mass conservation) forces $\lim _{t \rightarrow 1} B(t)=1$. This happens for pretty much the same reasons as in the single-boundary case.

Proposition A.4. For any solution of (A.19) (where $\rho_{B C}$ satisfies the heat equation and mass conservation) with the above stated initial conditions,

$$
\lim _{t \rightarrow 1} B(t)=1 \text {. }
$$


Proof. Neglecting the integral in (A.19) yields the inequality

$$
t+\frac{1}{2} \geq 2 B-\frac{1}{2} B^{2}
$$

which implies $B(t) \leq 1$ for $t \leq 1$. As for the complementary bound, let us reexamine (A.19) with the idea in mind to bound $\int x \rho_{B C} d x$ from below. It is noted that if we create an artificial density by shifting mass to the left, we certainly get a lower bound. However, let us remember that $\rho_{B C}$ is known to satisfy a few constraints. First, the total mass is $1-B$. Second, $\rho_{B C}$ is, in general, less than 1 and, using previous arguments that bound the dynamic density with a static one, in the region $x \leq 1, \rho_{B C}$ is less than $x$. Evidently, we may write

$$
\frac{3}{2}-t-\frac{1}{2} B^{2}=\int_{B}^{C} x \rho_{B C} d x \geq \int_{B}^{1} x^{2} d x+\int_{1}^{J} x d x,
$$

where the upper limit $J$ is given by $J=1+\frac{1}{2}(1-B)^{2}$. Replacing $x$ by 1 in the final integral in (A.21), we get

$$
1-t \geq \frac{1}{3}(1-B)^{3}
$$

which implies the desired result.

For our purposes, an important consequence of the analysis of the singleboundary problem is the implication, in the two-boundary cases, that the boundaries end up being smooth. This amounts to a corollary to Theorem A.3 and Proposition A.4.

Corollary. Consider the two-boundary Stefan problem as described, for example, in A.19 (with the above-mentioned initial condition). Then, if $T<1$, $B(t)$ and $C(t)$ are Hölder continuous on $[0, T]$.

PRoof. As a consequence of Proposition A.4 (and its reflection under the symmetry of the boundary conditions and the initial condition) for all $t<1$, the line $x=1$ stays in the interior region $B<x<C$. Now in the space-time interior region: $B(t)<x<C(t), 0<t<1$, it turns out that the solution $\rho_{B C}$ is in fact $C^{\infty}$. This follows directly from the observation that the heat operator $\partial / \partial t-\partial^{2} / \partial x^{2}$ is hypoelliptic and an application of Corollary 4.1.2 in [6]. Thus, focusing attention on $B(t)$, it is seen that $\forall t<1$ we can use the density $\rho_{B C}(1, t)$ as boundary data for the region $B(t) \leq x \leq 1$. It is easily checked that the density $\rho_{B C}(1, t)$ satisfies all the requirements for a $\rho_{F}(t)$-in particular, the fact that $\int_{0}^{1} \rho_{B C}(1, t) d t=\frac{1}{2}$ has now been forced by the fact from Proposition A.4 that $B(t) \rightarrow 1$. Evidently, Theorem A.3 tells us that the "unique" [for fixed $\rho_{B C}(1, t)$ ] solution has a smooth boundary. Similar considerations hold on the other side.

Our final result may now be stated as a simple proposition.

Proposition A.5. Consider the two-boundary Stefan problem in $[0,2] \times$ $[0,1)$ with the initial condition that the density vanishes if $x$ is less than 1 and 
is unity if $x$ is greater than 1 . The boundary $B(t)$ at which the density must vanish starts out at $x=0$, while $C(t)$, where the density goes to 1 , starts off at $x=2$. Stefan's condition is satisfied on both sides with the same numerical constant (of unity). Then there is exactly one solution to this problem and this solution is classical. Furthermore, for all $t \leq 1$, the initial symmetry is respected, for example, $\rho(x, t)=1-\rho(2-x, t)$. In particular, $B(t)=2-$ $C(t)$ and is given by the solution to the single-boundary problem on $[0,1] \times$ $[1,0)$ with $\rho_{F}(t) \equiv \frac{1}{2}$.

Proof. This follows from what is essentially a recapitulation of the proof of Theorem A.3. Consider the solution $\left(B, C, \rho_{B C}\right)$. We know that $B$ and $C$ are smooth [and that they collide at the space-time point $(1,1)$ ]. The smoothness will enable us to deploy a purely classical argument.

For a generic two-boundary problem with $D(t) \leq x \leq E(t)$, the same initial condition as the Stefan problem and analogous boundary conditions at $D$ and $E$-assumed to be smooth-let $\Phi_{D}^{[E]}$ denote the outward flux at the $D$ boundary and let $\Xi_{E}^{[D]}$ denote the inward flux at the $E$ boundary.

We will follow closely the routine of Theorem A.3, first defining an outer sequence of approximate solutions $\left(L_{k}, R_{k}, \rho_{L_{k} R_{k}}\right)$, where, for each $k, L_{k+1}=$ $\Phi_{L_{k}}^{\left[R_{k}\right]}$ and $2-R_{k+1}=\Xi_{R_{k}}^{\left[L_{k}\right]}$ ( $\equiv \Phi_{L_{k}}^{\left[R_{k}\right]}$ by symmetry). Obviously, the $L_{k}$ are increasing, the $R_{k}$ are decreasing and they have bounded derivatives on $[0, T]$ for any $T<1$ and so on; all this is just (a doubling of) Theorem A.3 in the special case where $\rho_{F} \equiv \frac{1}{2}$.

We note that, for all $k, B \geq L_{k}$ and $C \leq R_{k}$. We will explicitly demonstrate this only in the first case; the other case follows the (symmetrically) identical argument. There is nothing to prove if $k=0$. Suppose it has been established that $B \geq L_{k}$ and $C \leq R_{k}$. If we compare the densities $\rho_{L_{k} C}$ and $\rho_{L_{k} R_{k}}$, we rapidly come to the conclusion that $\rho_{L_{k} R_{k}} \leq \rho_{L_{k} C}$ and, since both vanish on $x=L_{k}, \Phi_{L_{k}}^{\left[R_{k}\right]} \leq \Phi_{L_{k}}^{[C]}$. It is therefore enough to show that $B \geq \Phi_{C}^{\left[R_{k}\right]}$. However, $\rho_{B C} \leq \rho_{L_{k} C}$, and hence, by another maximum principle argument, $\Xi_{C}^{[B]} \equiv$ $2-C \geq \Xi_{C}^{\left[L_{k}\right]}$. But

$$
\int_{L_{k}}^{C} \rho_{L_{k} C} d x+\Phi_{L_{k}}^{[C]}=1+\Xi_{C}^{\left[L_{k}\right]}-(2-C),
$$

while

$$
\int_{B}^{C} \rho_{B C} d x+B=1
$$

and thus it follows that $B \geq \Phi_{L_{k}}^{\left[R_{k}\right]}$. Evidently, when the limit $\left(L, R, \rho_{L R}\right)$ emerges, we will have $L \leq B$ and $R \geq C$.

As for the "inner" sequence, the only cause for pause concerns the choice of a startup function. With only a brief discussion, we will settle on the choice $A(t)=t$. (Notice this is just the bound we used in the particle system that goes back to Proposition 3.3 and the similar version that appeared as part of Theorem 4.1. Thus, we may justifiably insert such a bound without further discussion by stating that we are only interested in $B$ 's and $C$ 's that come 
from particle systems and hence a priori satisfy this inequality. However, for the sake of keeping this appendix self-contained, we will argue directly from first principles.) By appealing to (A.19) we see that the integral is degraded if as much mass as possible is pushed to the right. Recalling conservation of mass and the fact that $\rho_{B C} \leq 1$, we arrive at the inequality

$$
B+(2-C)-B(2-C) \leq t,
$$

from which the useful bounds $B \leq t$ and $(2-C) \leq t$ follow.

Starting then with $U_{0}=A(t), V_{0}=2-A(t)$, we define the successive outer approximations as before: selecting $T<1$, we define, for example, $t_{k}^{*}=\min \left\{t \in(0,1) \mid \Phi_{U_{k}}^{\left[V_{k}\right]}(t)=U_{k}(t)\right\}$, and set $U_{k+1}=U_{k}$ if $t \geq t_{k}^{*}$ and $U_{k+1}=$ $\Phi_{U_{k}}^{\left[V_{k}\right]}$ if $t \leq t_{k}^{*}$. (Of course, we will ignore the resetting altogether when the $t_{k}^{*}$ get beyond $T$.) It is noted that on $[0, T]$ with $T<1$, the function $A(t)=t$ satisfies the bounds $\dot{A} \leq 1 /(1-A)$ and $\dot{A} \geq(1-T) /(1-A)$ and thus may be substituted directly into the statement and proof of Lemma A.2. Thus, the ultimate limiting functions are seen to be Hölder continuous and, obviously, they are equal to the old $U$ (and $2-U$ ) in the case when $\rho_{F} \equiv \frac{1}{2}$. Now, all that is left is to show that $\forall k, B \leq U_{k}$ and $C \geq V_{k}$. Assuming this is true for some $k$, let us attend to the case $k+1$. Clearly, we are fine if $t \geq t_{k}^{*}$. If $t \leq t_{k}^{*}$, we run the same argument (in reverse) that we just used for the case of the outer sequence: for example, on the left side, it is seen that $\Phi_{U_{k}}^{\left[V_{k}\right]} \geq \Phi_{U_{k}}^{[C]}$, but $\Phi_{U_{k}}^{[C]}+\int \rho_{U_{k} C}=1+\Xi_{C}^{\left[U_{k}\right]}-(2-C)$. However, $\Xi_{C}^{\left[U_{k}\right]} \geq \Xi_{C}^{[B]}=\left(2^{U_{k}}-C\right)$ and $\rho_{U_{k} C} \leq \rho_{B C}$, so we get $\Phi_{U_{k}}^{[C]} \geq B$ as needed.

Now, examining the pair of limiting solutions, $\left(U, V, \rho_{U V}\right)$ and $\left(L, R, \rho_{L R}\right)$, we realize that $\left(U, \rho_{U V}\right)$ and $\left(L, \rho_{L R}\right)$ (restricted to $\left.x \leq 1\right)$ are both the unique solution to the single-boundary problem with $\rho_{F} \equiv \frac{1}{2}$. This pins down $B$, and likewise for $C$.

REMARK. The above analysis can be extended to a reasonable class of initial conditions with symmetric data. However, the use of symmetry in our argument is essential and we do not, at this time, see an argument where this can be circumvented.

Acknowledgments. The authors would like to thank D. Holley for having helped us in getting this project underway. We also thank M. Crandall, A. Friedman and J. Langer for useful conversations. In particular, we wish to thank J. Ralston for pointing out the hypoelliptic property of the diffusion operator and for various other discussions concerning the onedimensional heat equation.

\section{REFERENCES}

[1] Cannon, J. R. (1984). The One-Dimensional Heat Equation. Addison-Wesley, Reading, MA.

[2] Chayes, L., Schonmann, R. H. and Swindle, G. (1995). Lifshitz' law for the volume of a two-dimensional droplet at zero temperature. J. Statist. Phys. 79 821-831.

[3] Di Benedetto, E. and Friedman, A. (1984). The ill-posed Hele-Shaw model and the Stefan problem for supercooled water. Trans. Amer. Math. Soc. 282 183-204. 
[4] Fasano, A. and Primicerio, M. (1977). General free-boundary problems for the heat equation I. J. Math. Anal. Appl. 57694.

[5] Galves, A., Kipnis, C., Marchioro, C. and Presutti, E. (1981). Nonequilibrium measures which exhibit a temperature gradient: study of a model. Comm. Math. Phys. 81 127-147.

[6] HöRmander, L. (1963). Linear Partial Differential Operators. Academic, New York.

[7] IsHII, H. (1981). On a certain estimate of the free boundary in the Stefan problem. J. Differential Equations 42 106-115.

[8] Lifshitz, I. M. (1962). Kinetics of ordering during second-order transitions. Phys. JETP 15 939-942.

[9] Liggett, T. M. (1973). A characterization of the invariant measures for an infinite particle system with interactions. Trans. Amer. Math. Soc. 179 433-453.

[10] LigGETT, T. M. (1973). A characterization of the invariant measures for an infinite particle system with interactions II. Trans. Amer. Math. Soc. 198 201-213.

[11] LiggetT, T. M. (1985). Interacting Particle Systems. Springer, New York.

[12] Rost, H. (1981). Nonequilibrium behavior of a many particle process: density profile and local equilibrium. Z. Wahrsch. Verw. Gebiete 58 41-43.

[13] SpoHn, H. (1993). Interface motion in models with stochastic dynamics. J. Statist. Phys. 71 1081-1131.

Department OF Mathematics

UNIVERSITY OF CALIFORNIA

Los ANGEles, CALIForNia 90024

E-mail: lchayes@math.ucla.edu
DEPARTMENT OF Statistics

and APPLIED PRoBability

UNIVERSITY OF CALIFORNIA

SANTA BARBARA, CALIFORNIA 93106

E-mail: swindle@bernoulli.ucsb.edu 